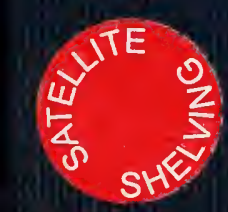




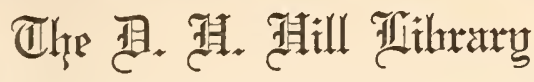

Xtarth Caralina State

Cillege

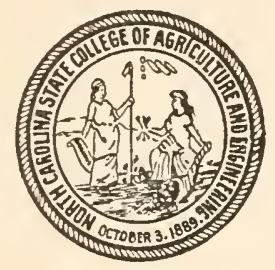

This book was presented Carnegie Institution QH4O6 554 
This BOOK may be kept out TWO WEEKS ONLY, and is subject to a fine of Mrris CENTS a day thereafter. It is due on the day indicated below:

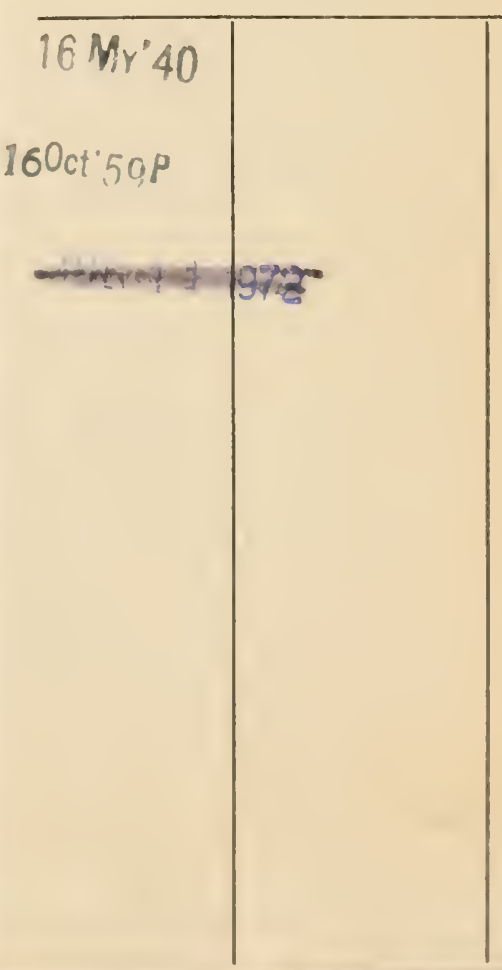




BY

GEORGE HARRISON SHULL

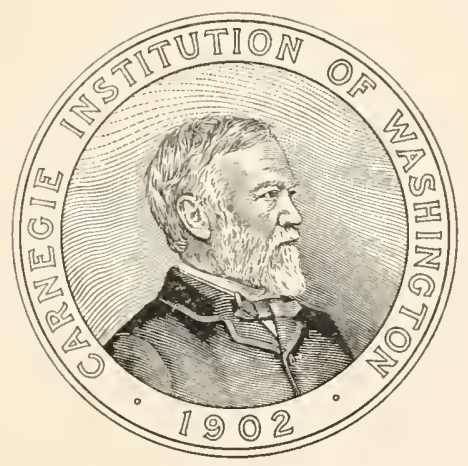

WASHINGTON, D. C.

Published by the Carnegie Institution of Washington.

I 909. 
Carnegie Institution of Washington Publication No. 112

Papers of the Station for Experimental Evolution No. 12

THE CORNMAN PRINTING COMPANY

CARLISLE, PENNSYLVANIA 


\title{
BURSA BURSA-PASTORIS AND BURSA HEEGERI: BIOTYPES AND HYBRIDS.
}

\author{
By George Harrison SHull.
}

\section{INTRODUCTION.}

Darwin recognized in the facts of variation a key to the riddle presented by the multiformity and many obvious interrelations of all living things. Since the appearance of the Origin of Species the observation and discussion of variations have assumed a dominant place in biology, and a serions conflict has recently developed regarding the interpretation of the observed facts. It is now generally recognized that this conflict can be brought to a termination only through the application of experimental methods. Inspection alone can not decide the question as to how an observed variation originated and what bearing it may have on the future of the race in which it occurs. Studies in the museum and in the ficld only discover the fact, and to some extent the range, of variation occurring under a more or less limited and inadequately known range of conditions, and can not certainly determine its cause or causes; neither can these means supply more than a suggestion based upon insecure inference as to the hereditary nature of any variation. The causes of variation can be determined only by subjecting equivalent material to different controlled conditions, and their hereditary relations can be learned only through the conduct of pedigreecultures.

We already know, as a result of experimental work in these directions, that variations are of fundamentally different types, having different causes and obeying different laws of development and heredity. A knowledge of these facts impresses an important principle, namely, that the range of applicability of any conclusion reached by the investigation of one class of material or one characteristic can be determined only by similar experiments with other material and other eharacteristies.

This has been one of the guiding principles in the institution of the experimental work at the Station for Experimental Evolution of the Carnegie Institution of Washington. Numerous species of plants and animals of quite wide relationships have been bronght under observation, and it may be expected that each will in time give material assistance in determining to what extent principles and hypotheses now available have general validity, and will also lead to the discovery of such new principles, or such modifi- 
cations of the old ones, as may be necessary for the interpretation of the observed facts of eroltition. Among the species now being studied, one that has very early yielded results of interest in this connection is the common shepherd's-purse of our dooryards, Bursa bursa-pastoris (L.) Britton.

Bursa (Capsella) bursa-pastoris is known to the taxonomist as an exceedingly variable species, which seems to have been brought to this country from Europe, but which is now naturalized and almost universally distributed throughout the North Temperate zone. So strikingly different, qualitatively, were the characters of different individuals observed growing side by side in nature that it was difficult to believe that they all belonged to a single series of fluctuating variations, and when the opportunity offered to make pedigree-cultures this species at once suggested itself as farorable material. This thought was based not alone upon its apparent polymorphism, but also upon its hardiness, ease of culture, and the impossibility of its having been subjected to any of the artificial conditions of isolation, crossing, etc., which are usually thought to render plants of economic value unfit to give information regarding the behavior of plants in nature.

No one has ever attempted to "improve" the shepherd's-purse, and athough its rapid extension over its present great range is undoubtedly dependent upon the agency of man, both in supplying suitable habitats and more directly in the transportation of the seeds, yet in performing these operations his work has been wholly nnintentional, and he is therefore to be classed with the other accidental agents of nature, thus leaving to Bursa the solution of its problems of maintenance, extension of range, and evolutionary progress, under conditions which are fundamentally like those that must be met by any other species in a state of nature.

These cultures were begun in April, 1905, and were continued until the spring of 1907, when they were temporarily abandoned becanse other investigations necessitated my absence from the Station for Experimental Evolution during rather extended periods, and thus made it impossible to continue the work advantageous1y on a species like Bursa, which shows no dependence upon the seasons, but blooms and fruits whenever external conditions are such as to make its development possible. It is hoped that these studies on Bursa may be continued in the not distant future, but as some of the conclusions arrived at have been already presented before several scientific bodies, ${ }^{*}$ it seems desirable to publish a more comprehensive account of the work than has been done up to this time, even though the evidence is in many places more or less fragmentary.

When undertaking such cultures with any new class of material much that is necessary for the most satisfactory and cconomic conduct of exper-

\footnotetext{
*Sections F and G, A. A. A. S., New York, December, Igo6; Seventh International Zoological Congress, Boston, August, 1907; Botanical Society of America, Chicago, January, rgos.
} 
iments must be learned by experience, for, as said before, mere inspection of an individual or of a single generation can not distinguish between important and unimportant variations until the pedigrees themselves furnish solutions to the question. For this reason there must be in the beginning a much more minute analysis of the material than is later found necessary or desirable. Most of the cultures which I have made thus far have been directed toward determining what variations are of the fluctuating kind and what are fully transmissible to the offspring.

In one group I have now had large numbers of plants of the fourth pedigreed generation and a few of the fifth under observation, and in a number of other cases the third generation has been extensively grown. I find that while certain variations which were selected disappear in the first or second generation, others remain constant, easily recognized differentiating marks which, except in one form, show no transgression of the characteristic features of any other form studied. These forms are, therefore, distinct elementary species, or biotypes, each characterized by certain constant features and each with its own normal range of fluctuating variability.

The systematist has not yet decicled what treatment to give to elementary species, and any nomenclatorial scheme must be regarded, therefore, as purely tentative; but utility can not wait for concerted action on the part of taxonomists in devising a suitable systematic designation of elementary species, and I have therefore for the sake of convenience assigned to these elementary forms of Bursa simple names which can be attached to the accepted specific name to form a trinomial. I was at first inclined to use binomial names which would leave the Limnean specific name, bursapastoris, as the valid name of the aggregation of elementary forms having the same general habit and the triangular or obcordate capsules. It might, then, be looked upon as a superspecific name which would remain just as useful in the everyday conversation and experience of the botanist as when the aggregation for which it stands was believed to be a $11 n$ it.

The fact that corresponding series of elementary species or biotypes may occur in different related species, as will be shown later in the discussion of the hecgeri hybrids, makes the trinomial much to be preferred, for corresponding forms may then be given the same name without confusion. Thus Bursa bursa-pastoris heteris and Bursa heegeri heteris may be used to denote two forms which are alike in rosette-characters but different in the capsule-character, the latter character being accepted as of specific value.

That Bursa bursa-pastoris is a composite species was first given public recognition by Lotsy (1906) about a year after my cultures were begun, and his statement did not come to my notice nntil after I had presented my first acconnt before the American Association for the Advancement of Science at its New York 1neeting, December, 1906. His statement is very brief, and the chief interest of his account consists in the photograplis 
which show samples of three families which bred true to rather strikingly distinct characters in his cultures. To these several forms he assigned trinomial names, though no adequate description is given. No statement is made as to the extent of the eultures upon which their standing as permanent biotypes is based, nor is there any statement as to the extent and nature of the fluctuating variability of each of the forms figured. Until attention is given to such matters by the student of pedigree-entures it will be impossible to properly estimate the security of conchusions drawn, or to recognize with any certainty the identity of biotypes discovered by different investigators.

To obviate these difficulties, I wrote to Dr. Lotsy for pedigreed seeds of his sereral types, in order that they might be grown beside my own for the purpose of testing their identity, but reeeived the reply that none were arailable. The questions as to whether Lotsy's names are therefore to be considered nomina muda and whether I am justified in assigning new names to the biotypes to be discussed below, which may or may not be identical with one or more of Lotsy's mamed elementary species, I leare to the taxonomist to decide. Indeed, in adopting the names used in this paper I have been gorerned entirely by the demands of present utility and not by any thonght that these names will be accepted by taxonomists as having proper standing in the nomenelatorial system now recognized by them.

Still more reeently, Almquist (1907) has published orer 70 named " elementary species" of B. bursa-pastoris, but these are described in rather general terms, and the cultures were conducted in the open garden unguarded, usually for but 2 to 4 generations. Almquist states (p. 5) that after this length of time the cultures had either died out or were no longer pure. He assumes that this "loss of purity" is due to vicinism with the other elementary species growing on neighboring plots, and some of it doubtless was. In my own enltures I have observed that some of the differentiating characters used by Almquist disappear upon eontinued breeding, even when ricinism is carefully excluded. It seems that members of the same biotype coming from different habitats may retain certain fluctuating differences, such as differences in texture of leaves, degree of redness of veins, degree of epinastic growth in the petioles, ete, for several generations of uniform treatment, but finally merge into identical forms, eren when crossing with other biotypes is entirely precluded by careful guarding.

While I can not say with certainty that any of Almquist's "elementary species" are not distinct and permanent types, my experience indicates emphatically that he has not demonstrated that they are. I think it rery probable that a number of the elementary speeies figured and deseribed by him will not stand the test of longer culture under more carefully controlled conditions. Howerer this may be, it seems evident that the number of elementary species of Bursa may be considerable. At the time of the first 
presentation of my studies on Bursa before the American Association for the Adrancement of Seience (December, 1906), I was pretty sure that I had demonstrated the existence of 11 biotypes, but the disappearance of one or two of these since, led me to reduce the number to 4 , as publisherl in the extract from that paper (Sihw11, 1907).

These four forms: whose distinetness and permanenee I have demonstrated beyond a possible etuestion, have been studied in their hybrid combinations, and it is the eultures of these and their hybrids with which the present paper will mainly deal, though some features of other cultures will be diserissed.

It is doubtful whether any bit of technique that has been recently added to the tools with which the biologist may operate in molocking the mysteries of protoplasmic organization is of so far-reaching importance as the process of hybridization. I say has been recently added becanse, although the art of producing hybrids is very old among the brecters of animals, and nearly 200 years old among gardeners, little of scientific valuc could be secured by means of hybridization nntil some of the fundamental laws involved in the process were recognized, and it is only within the last eight years that biologists have gained sufficient insight into the belavior of hybrids to give interpretations of the results of hybridization any value as indications of protoplasmic structure and behavior.

Two general types of hybrids are readily recognizable, namely, the constant and the splitting. The former may or may not give indication of the characteristics of their parents, being usually, but not alvays, intermecliate between the parents in nost of their characters. The splitting hybrid always indicates by its offspring what were the characteristics of its inmediate ancestors. It is the latter type of hybrid which is of the greatest 11sefulness in giving insight into the structure of the germ-plasm. Hybridization in such cases does not only serve to unite in the same individual all of the characteristics of both parents, but as successive generations are followed, it results in a complete analysis of all the points of difference existing in the two biotypes between which the cross was made.

The conduct of such analysis by hybridization is particularly simple in the case of plants which, like Bursa, readily self-fertilize, because once the first-generation hybrid is sceured the process of analysis goes on generation after generation, until all the allelomorphic differences of the parents are made manifest by beins separated in a pure state in different individuals.

In some respects even more sitisfactory evidence of the clenentary character of two forms is to be derived from their behavior when crossed than from their conduct in straight pedigrees. If, for instance, two forms sup)posed to be elementary to each other should be, instead, merely extreme fluctuants of a single biotype, their cross-bred progeny would sliow a fluetuating series, including perhaps the two parental extrenes, but coutl harotly 
be expected to produce a constant intermediate progeny, nor one which would show the two parental conditions in an expected Mendelian ratio. Occasionally in the conrse of pedigree-cultures some slight difference in treatment will produce a modification in some progeny, which may lead to cloubt as to its classification, and perhaps even after sereral generations this doubt will not be wholly removed. In such cases hybridization is almost certain to give decisive results, as there often appears to be a more characteristic development of morphological features in the hybrids than in the pure-bred strains. This may be due to the fact that the allelomorphs are bronght into different relations with other allelomorphs in the recombinations which take place in the second and later generations, thus eliminating any inhibiting or modifying influence which may hare resulted from the constant presence of some particular allelomorph or combination of allelomorphs, or of some permanent conclition of the cytoplasm, in the pure strain. This elimination of modifying factors would allow a more accurate definition of the unit-characters involved. Or, on the other hand, the better development of characters in the hybrids may be dute to the fact, long recognized, that heterozygosis produces a stimulation which increases regetative rigor, and this should have the same effect as good cultural conditions in bringing all characters to their full typical development.

\section{MATERIAL AND METHODS.}

For the beginning of the cultures, seeds were secured from a number of more or less strikingly different individuals growing in the vicinity of Cold Spring Harbor, Long Island; Chicago, Illinois; New Carlisle, Ohio; Manhattan, Kansas; and Tucson, Arizona. Leares of each of the plants chosen as parents for the cultures were carefully preserved, in order that the conditions of the offspring might be compared with those of the parent and, in each pedigreed family raised since, care has been taken to preserve in the herbarium samples of every variation of sufficient magnitude to strike the eye.

From 20 lots of seeds of Bursa bursa-pastoris brought in from nature and 1 lot of $B$. heegeri reecived by Dr. MacDottgal from Professor SolmsLaubach, I have now had under observation about 200 pedigreed fanilies, including something over 26,900 individuals of Bursa bursa-pastoris, 2 small families of Bursa heegeri, and 5 families representing reciprocal crosses between these two species and involving over 2,500 individuals.

All of my cultures have been carried on under glass, the first 7 montlus in the sky-lighted rom of the laboratory at the Station for Experimental Erolution, and since that time, $i$. e., after December 7,1905 , in the glass propagating-house at the same place. It was found that the light was too clim in the former room to bring out the characteristic features of the plants and to keep them in a state of vigorous health. The leares became more 
elongated than is normal, and the lobing more shallow and therefore less characteristic, so that it was less easy to estimate the uniformity and distinetness of the several pedigrees than when they were grown under more farorable conditions. Even specimens belonging to the most deeply and distinctly lobed families, when grown in the darker portions of the room, retained their unlobed, juvenile type of leaf throughout life, sending up a weak flower-stem from the juvenile rosette, and in such cases the relationships could not be recognized, sinee the early leaves of all the forms studied are very similar. The same difficulty was also experienced in the propagating-house when the menibers of certain families were allowed to remain too long crowded in the seed-pans. The offspring of these juvenile plants have not been extensively studied, but from several families evidence has accumulated which indicates that when again given favorable conditions the offspring of these characterless plants return to the characters of the family from which they sprang.

In all cases the seeds have been sown in soil sterilized in an autoclave in the manner usually adopted by students of pedigree-cultures, and the efficiency of the method is inferable from the fact that in all these cultures only 3 seedlings occurred whose origin was unknown. These unexpected seedlings were an Oralis, a Mollugo, and an Erechtites, and it does not seem likely that any of these withstood the long-continned high temperature of the antoclare, but rather that they were blown through the ventilators of the propagating-house in a heavy wind-storm.

It was soon demonstrated that Bursa has many features which make it advantageons material from a technical point of view for pedigree-culture work. The habit of the plant, consisting as it does of a moderately lax rosette and nearly naked, erect flower-stem, allows the preservation of the rosettes as herbarium specimens whose characters are almost as easily studied as are those of the living plants, and the inflorescence may be corered with paraffin-paper bags to prevent chance erosses with other specimens without appreciably interfering with the photosynthetic work upon which the healthy development of the plant depends.

The small size of the plants makes it possible to raise them to maturity in 3-inch pots in many of the forms, though it is 11sually found advantageous to repot to 4 -inch pots those which it is clesired to keep for seed. This quality allows a large number of specimens to be raised in a small compass.

In most instances the life-cyele is short, reculiring only 3 to 4 months between the sorving of the seed and the gathering of the ripe seect of the earliest matured individuals. In several of the forms of P. Inuse-pastoris, and in $B$. heegeri, however, 8 to 9 months were needed. The seeds germinate in 5 to 8 days without a period of rest, thus making it possible to accumulate data from a number of sucessive generations in a short time. 
The flowers are adapted to both cross- and self-fertilization, all the evidence now at hand indicating that the latter method is normally by far the more efficient. Leaving out of account 2 of the 21 original cultures which showed by their constitution that their parents were hybrids, less than 1 per cent of the plants raised from seed collected in nature shorred evidence of being the result of cross-fertilization between different biotypes. Crosses between different flowers of the same plant and between different indivichals of the same biotype may take place somewhat more frequently than crosses between the flowers of plants belonging to different biotypes, thongh nothing in the resultant offspring gives any clue to the frequency of such crosses.

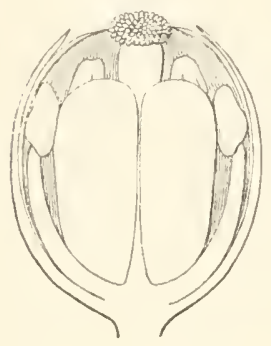

A

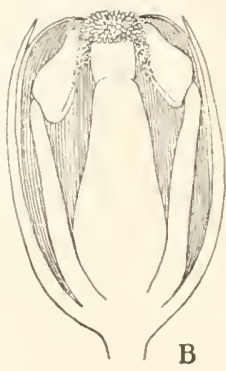

B

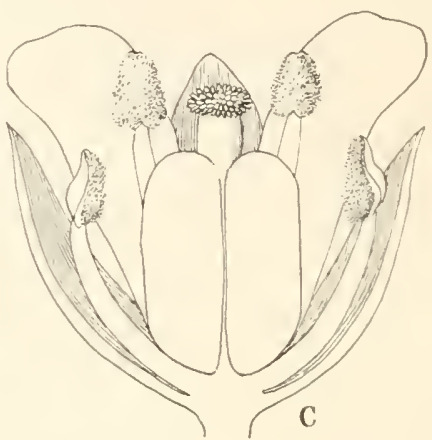

FIG. 1.-Enlarged sections of buds and flower of Bursa bursa pastoris, showing three stages in anthesis. A. Exposure of the stigmatic surface for the reception of foreign pollen. B. Anthers opening in contact with the stigma, thus insuring self-pollination. C. The flower fully open, allowing the access of the visiting insects to the pollen. All magnified 20 diameters.

The conditions which faror cross-fertilization are: (a) Slight proterogyny, which allows the stigma to receive foreign pollen some hours before the anthers of the same flowers delisce. The distal portion of the globular or disk-shaped stigma is exposed between the tips of the unopened sepals and petals (fig. 1, A). (b) Although the fully dereloped inflorescence is a typical raceme, the flowers and buds are arranged in a nearly flat-topped corymb having the flowers at the ciremmerence, giving this part of the inflorescence a condition quite analogous to that of the head of the Compositx. in which the whole inflorescence appears to act the part of a single flower in the attraction of insects. Small insects, particularly flies and small bees, visit the flowers freely. These rest mpon the top of the inflorescence as a whole while visiting the several individual open flowers about the circumference. In these fully open flowers forming the exterior circle of the infloreseence the anthers hare opened, while in the second circle the smmmits of the stigmas are exposed on the same general level as the rest of the corymb. As the insect walks about orer the top of the inflorescence, the 
chances appear favorable for the lodgment of pollen from the outer circle of flowers, or from the flowers of other plants, upon the exposed stigma-tops of the second circle.

Adaptation to self-fertilization is found in the facts: $(a)$ that the stigma is also receptive on its under (proximal) surface as well as on the upper (distal) surface, and (b) that the anthers delisce while they are still held in contact with this under surface of the stigma by the erect segments of the perianth (fig. 1, B). Soon after the anthers open the petals begin to spread, thus exposing the pollen to be carried away to other flowers by visiting insects (fig. 1, c).

At the beginning of these experiments paraffin-paper bags of suitable size were not available, and as inseets were not abundant in the room used for the cultures, the only precaution taken to guard against cross-pollination was to set the flowering specimens intended as seed-plants somewhat apart from each other and from other flowering specimens. As will appear later, a few individuals, of unexpected character, are doubtless to be attributed to this unguarded condition of the earlicr cultures, but it will also be seen that the percentage of such chance crosses is extremely small. As soon as possible, suitable paper bags were secured, and since then the enttures, with few exceptions, have been carefully guarded.

Although crossing among the unguarded cultures in the greenhouse has been of rare occurrence, it was evidently more frequent in the material secured from nature. Of the 21 original cultures 2 proved to be of hybrid origin, while a fraction of 1 per cent of the rest indicated by their atypic condition that they were probably the restult of cross-pollination. Only when the pollen comes from some form which is dominant to the pistilparent is the fact that a cross has taken place obvious in the first generation. It seems fair to assume that on the average as many crosses take place with a recessive pollen-parent as with a dominant, and this assumption would require that hybridizations occur with twice the frequency with which they become obvious in the $F_{1}$ offspring. On this basis the frequency of cross-pollination between different biotypes of Bursa in nature, as indicated by these cultures, is about 1 to 65 as compared with the frequency of self-pollination and crosses between flowers of the same plant or between plants of the same biotype. Of course this ratio is based upon a very limited number of specimens and can be expected to vary greatly in different lots of material of the same magnitude, but at least the great preponderance of self-fertilization may be safely inferred in this species when in a state of nature.

These characteristics of Bursa which make the production of pure selffertilized lines easy are opposed to the ease with which cross-fertilization may be controlled. The fact that self-fertilization takes place before the petals spread makes it necessary to carefully remove the stamens about a 
day before the buds open. As the buds are at that time quite small, the technique of cross-pollination is somewhat delicate. However, with a needle-pointed forceps the calyx, corolla, and stamens may be readily cut away from about the young pistil, and the eye and hand soon become so skilled that the work can be done swiftly and with as much accuracy as may be attained in the castration of a lily.

\section{PURE CULTURES OF BURSA BURSA-PASTORIS.}

The four biotypes of Bursa bursa-pastoris with which this paper mainly deals are distinguished from each other by certain charaeteristic lobings of the leaves. For convenience I have assigned to them the names Bursa bursa-pastoris heteris, B. bp. temuis, B. bp. rhomboidea, and B. bp. simplex. It has been impossible to determine which of Almquist's "elementary species" agree with these, but it is almost certain that he has assigned names to several fituctuations of the same biotype. If this is true my four types are more inclusive than his and under my names will need to be grotped a number of forms which he has considered distinct elementary species.

\section{Bursa bursa-pastoris heteris $\mathrm{n}$. sp. element.}

Plants belonging to this biotype have the leaves divided to the midrib, the terminal lobe being usually separated from the nearest lateral lobes by deep, clean-cut incisions. The lateral lobes consist essentially of two features-an elongated, attentrate portion which I call the " primary lobe," and a more or less rounded or angular portion which forms a " secondary lobe" in the distal axil of the primary lobe (fig. 2). As in the characterizations of all the following forms, this description refers to the climax-leaves of the rosettes in properly grown specimens, since the juvenile leaves of all the elementary species of Bursa are entirely unlobed, and starved or crowded specimens of all the forms may reach maturity with only jurenile leaves, as already stated. Several of Almquist's recently described forms would obvionsly range themselves under this description, though it is always possible, of course, that forms which possess the described characteristics may have still other permanent characteristics which would render them distinct elementary species.

The first of the forms recognized by Almquist which can certainly be placed here is his Capsella bp. mbella (see his figs. 5 and 6). His C. bp. angustiloba (his figs. 56 and 57) represents the most pronounced development of this type, and others which probably belong here are reuteri (fig. 11), grandiflora (figs. 12 and 13), hicmalis (fig. 20), grossa (fig. 22), autumnalis (fig. 24), segetum (fig. 28), wittrockii (fig. 44), rhombea (fig. 48), rhombclla (fig. 53), cllipsoidea (fig. 55), and fucom (fig. 60).

My first pure-bred family of $B$. bp. heteris (pedigree-number 040.3) was grown from seeds collected by J. Marion Shull at Edgewood, New Carlisle, 


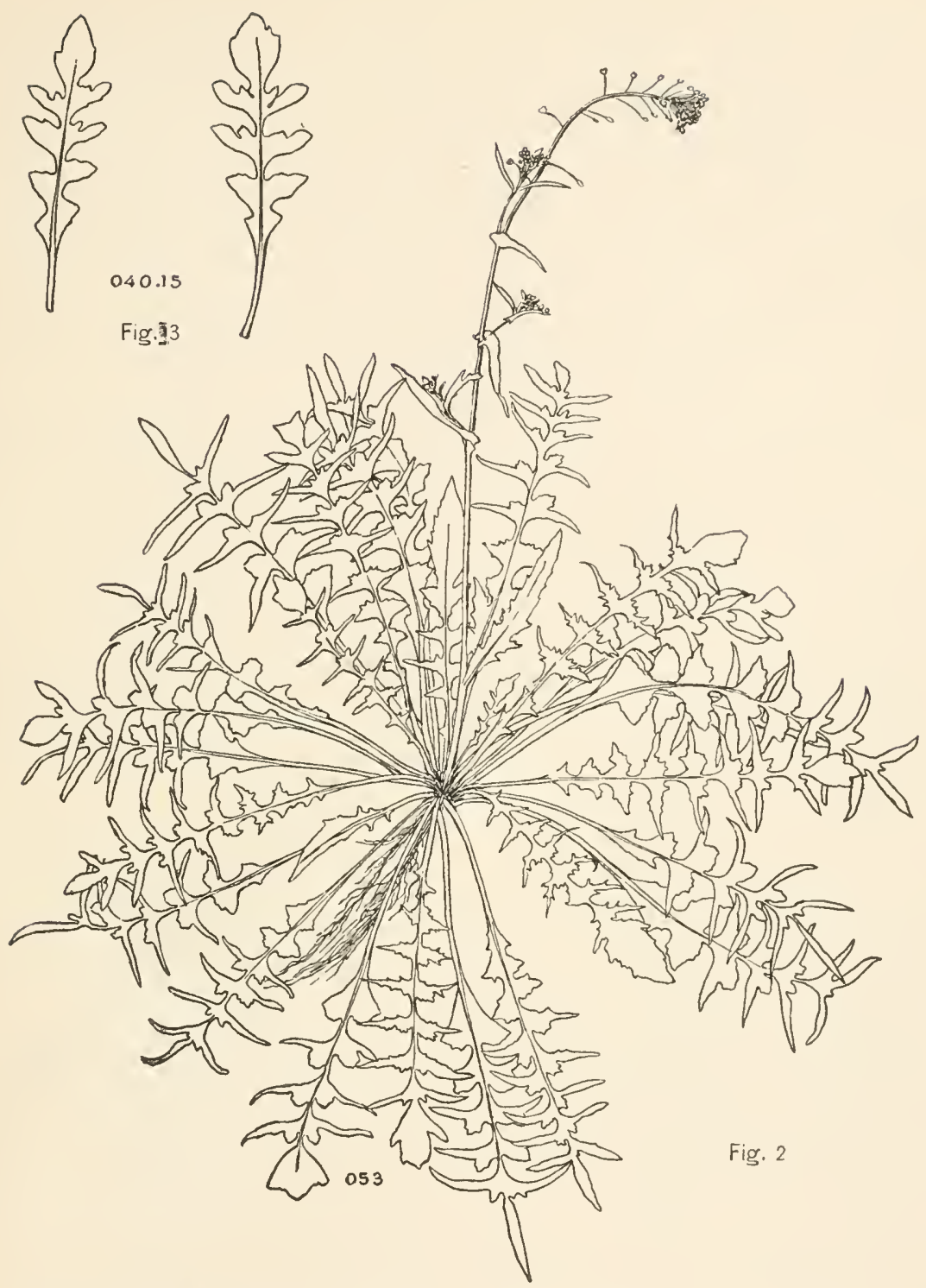

FIG. 2.-Bursa bursa-pastoris heteris. A typical specimen of my first pure culture of this biotype.

FIG. 3.-Bursa bursa-pastoris heteris. Climax leaves of the rosette of a specimen growing in nature in Jackson Park, Chicago. 
Ohio, May 28 and June 2, 1905. The climax leaves of the parent were of the most pronounced heteris form. From seeds sown June 26, 1905, 39 specimens were grown, all of which agreed with the parent and with each other in the character of lobes as described, though there was some fluctuation in general aspect due to difference in elongation of the rachis by means of which the lobes were more or less crowded, some variation in the attenuation of the primary lobes, in the degree of lobation of the upper rosette leares, in the angle which the rosette leaves made with the horizontal, and in the prominence of the rounded secondary lobe. This secondary lobe was characteristic, however, and in not a single individual was it absent in the climax leaves of the rosette.

The significance of several of these variations was tested by using their best representatives as parents of a second generation. From 3 of these $(053.30,053.31,053.32)$ have been raised 839 speeimens, all but the 54 offspring of one individual being strictly like the grandparental and parental form. The one aberrant family $(053.30)$, the parent of which had the upper leaves of the rosette less deeply lobed than usual, showed complete suppression of lobes in 5 individuals, and in the remaining 33 which were noted the primary lobes were broader than usual and the secondary lobes less prominent. It is desirable to test the possibility that these characteristic secondary lobes may be rendered eompletely latent by selection, and, if this is possible, to discover by what means they may be again made manifest.

At least two other pure-bred cultures coming in from nature belong to B. bp. heteris, but were considered for two generations to represent two very distinct elementary forms. It has been the striking modifications observed in these two pedigrees which have led me to suspend judgment on all my cultures except those whose behavior in hybrid combinations has left no possible question as to their distinctness and permanenee.

The basis for the assmmption that these two pedigres represented distinct elementary species was the facts that the aspect of each of these families was very different from that of any other culture and that there was sreat uniformity among the individuals belonging to either single family, $i$. $\ell$, while there was a strong break between the families there was almost no variation within the family. This was presnmably the basis of Almquist's estimates as to the distinctness of his forms, and the following account of these two families supports my attitude of doubt as to the soundness of his results.

040.15: This speeimen was found growing in Jackson Park, Chicago, by Charles A. Shull, who collected the seeds in the summer of 1905. Climax leaves taken from the rosette show only a sliglit development of the heteris characters as described above (fig. 3). Though the sinuses reached the midrib, the primary lobes were not sharply attenuate, and the secondary lobes, 


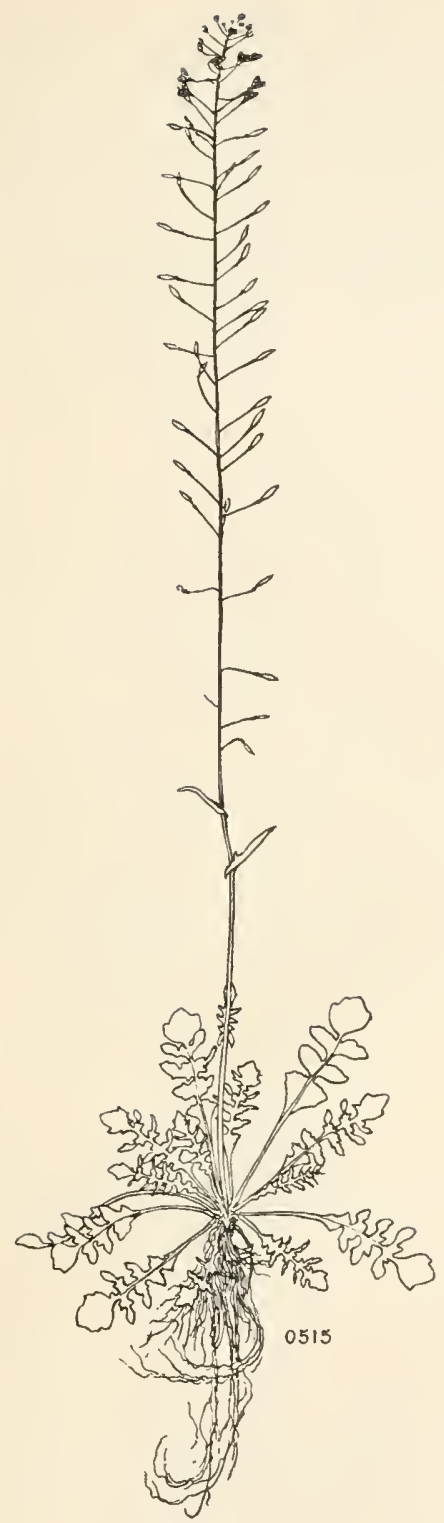

Fig. 4.

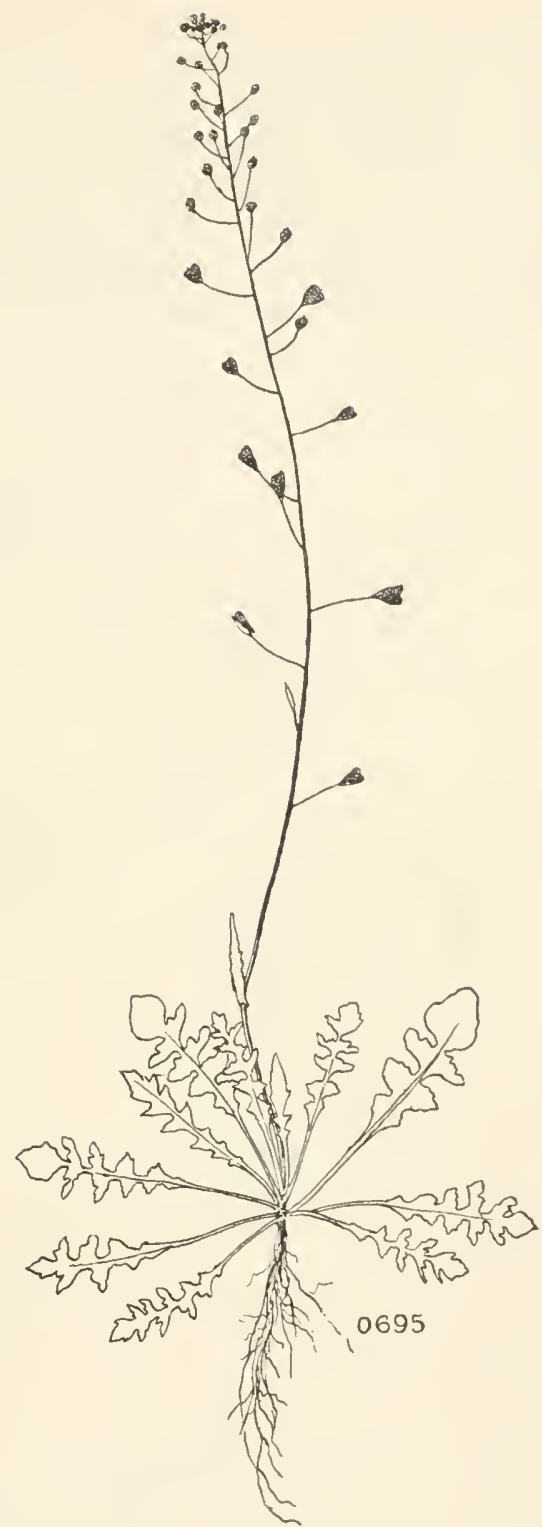

Fig. 5.

FIG. 4.-Bursa bursa-pastoris heteris. Typical specimen from offspring of plant shown in fig. 3 .

FIG. 5.-Bursa bursa-pastoris heteris. Offspring of sib of plant shown in fig. 4 . 
though present, were not strongly marked. The sceds were sown in the propagating-house December 27, 1905, and produced 106 offspring, all of which had a pectliar grayish aspect, owing to the fact that the leaves were freely dotted with small specks of red. The rosettes were very lax, the relatively few leaves made a rather wide angle with the horizontal, and the leares, while possessing both the primary and secondary lobes, had the latter small and angtular and little more marked than serrations borne by the primary lobe, which all experience ttp to the present time indicates are of only fluctuating value. (See fig. 4.) All had simple slender stems which came to bloom in 3 to 4 months from the time the seeds were sown as compared with 8 months required by the above-described family of typical $B$. bp. heteris.

Self-fertilized seeds of two of these plants (0515.95 and 0515.96) were sown in May, 1906, and gave uniform progenies, but having an aspect quite different from that of the parent generation just deseribed. Although the rosettes in this second generation were strongly ascending and were fewleared, as in the preceding generation, the texture of the leaves was much less firm, the grayish aspect was wholly lost, owing to the absence of the red specks, and secondary lobing was also much reduced, giving a condition resembling the grandparent as it grew in nature (ef. figs. 3 and 5). The conditions in the propagating-hotse during the development of these families were unsatisfactory, and their great change from the characteristics of the preceding generation did not shake my faith in their distinctness from my other types, since within the family there was still great uniformity, seeming thus to demonstrate that whatever differences were observed were due to the differences in environment during the time of development of these two generations.

An average specimen from one of these two families was chosen as the parent of a third generation. The pollination of this plant (0695.158) was unguarded, but it was grown well separated from all other Bursa cultures. The sceds were sown on November 1, 1906, and prodttced 213 plants, all of which possessed well-marked the characteristics of $B$. bp. heteris, having completely lost all the peculiarities which had led me to believe that this pedigree belonged to a distinct biotype. (See fig. 6.) Other proofs that 040.15 was a specimen of $B . b p$. heteris were derived from hybrid families formed by crossing its offspring with plants belonging to other biotypes. Except in one case only the $F_{1}$ hybrids from these crosses have been studied and they will not be considered in detail at this time, but these firstgeneration hybrids showed the characteristics which they should have possessed if typical B. bp. heteris had been used. The one $F_{2}$ family (0693.203) which has been reared from these hybrids will be considered later. (See p. 42.) 
050.80: Another original culture which now seems to belong to $B . b p$. heteris was the offspring of a very robust plant collected in a dooryard near Cold Spring Harbor, Long Island, April, 1906. This plant was taken up and potted in the greenhouse, where its pollination was guarcled. The earlier leaves of this plant were recognized as resembling B. bp. heteris, except in the less sharp attenuation of the primary lobes, but later leavesthe climax-leares-were large, and, in addition to the secondary lobes characteristic of $B . b p$. heteris, they had somewhat quadrangular secondary

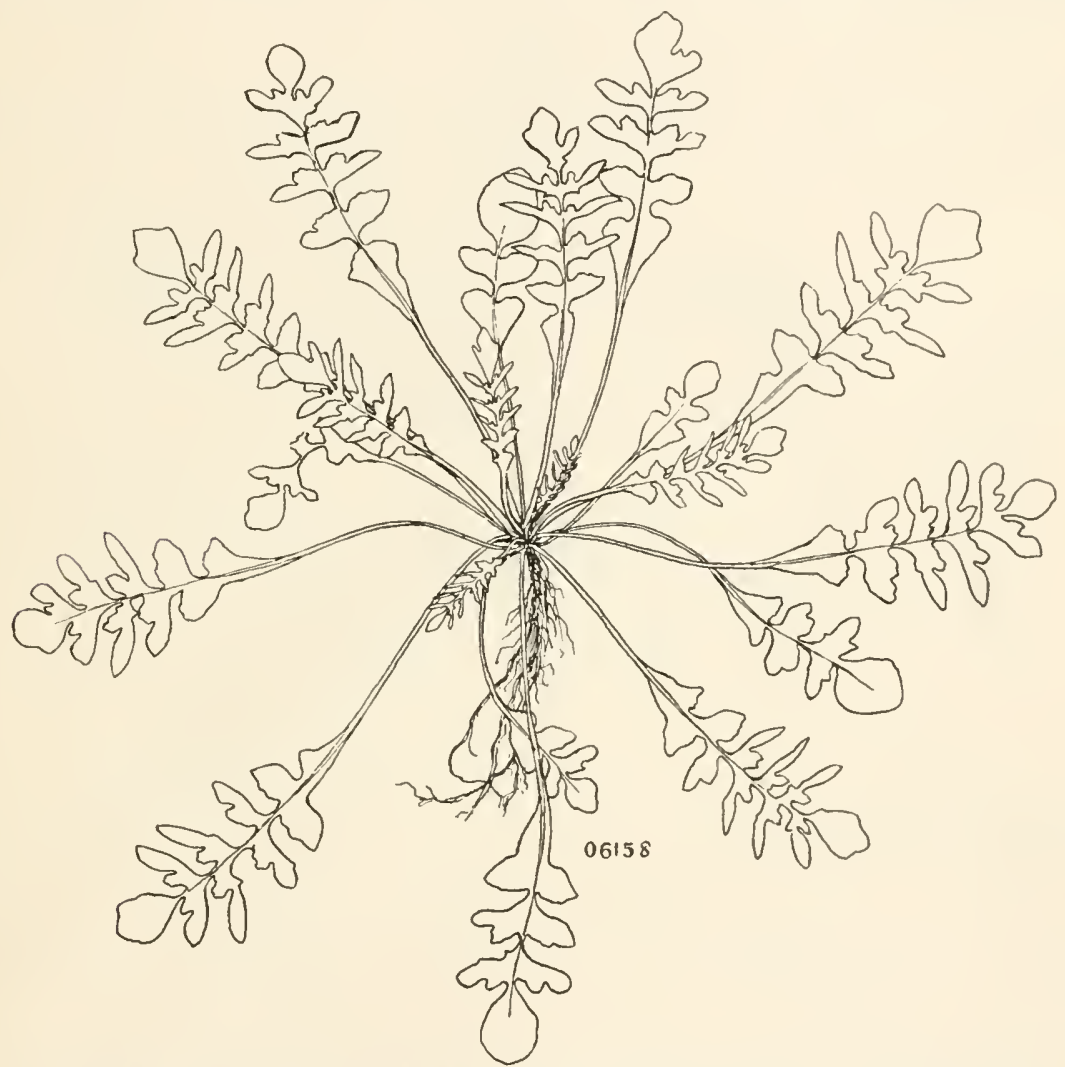

F1G. 6.-Bursa bursa-pastoris heteris. Offspring of sib of plant shown in fig. $\pi$.

lobes in the proximal axils of the primary lobes, and these square lobes were sometimes almost cut off from the primary lobes, giving the leaf the peculiar form usually described as interruptedly pinnate (fig. 7). The seeds of this plant were sown May 23, 1906, and produced 352 offspring. These were badly damaged by the thrips, 81 being killed and 57 so stunted as to make an estimation of their characters uncertain. The remaining 214 formed a consistent group unlike its parent and also unlike any other 
of my cultures. Few showed even a suggestion of the squarish lobes which rere so conspicuous in the parent, thus indicating that the strong development of that characteristic in the parent was in all probability merely a fluctuation. These plants differed from those considered typical of $B$. bp. heteris in having the primary lobes of the climax-leaves oblong and blunt, not attenuate. However, in some specimens the later leaves showed the attenuate lobes of typical $B$. bp. heteris, and this fact leaves little doubt that another generation would have completely demonstrated that this family belongs to $B$. bp. heteris.

Whether a culture (06+5) produced from seeds sent by Dr. D. T. MacDongal, from Tucson, Arizona, is likewise identical with the $B$. bp. heteris grown from seeds collected in Illinois, Ohio, and Long Island, has not been sufficiently tested. While the Tucson plants had in a most strongly

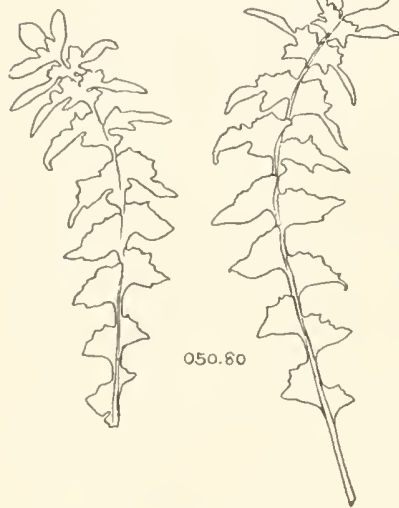

F1G. 7.-Bursa bursa-pastoris heteris. Climax leaves of a specimen growing near Cold Spring Harbor, Long Island. marked way the primary and secondary lobes described above, there were striking differences in the texture and color of the leaves, the form of the lobes, and the form, size, and texture of the stem-leaves or bracts (fig. 8). As only one generation of plants has been grown from these seeds, no proper grounds exist for attempting to decide as to the permanence of the differences exhibited, but it might be expected that plants from the hot, dry, intensely lighted plains of Arizona would display considerable changes of a purely transitory nature on being transferred to the moist atmosphere and relatively dim light of a more northern propagating-house.

Field observation indicates that the type of rosette possessed by Bursa at Tucson, of which this culture was a fair example, is the common type if not the only type of rosette displayed by Bursa in that locality and westward. Many facts now at hand suggest that the heteris type is the primitive type of rosette from which the forms to be deseribed below have been derived, and the relatively wide geographical distribution of this type is in strong support of this view.

Bursa bursa-pastoris tenuis $\mathrm{n} . \mathrm{sp}$. element.

This differs from the preceding type in several important features. The sinuses are relatively shallow, rarely extending nearly to the midrib in very strongly developed individuals. The terminal lobe is not separated from the nearest lateral lobes by deep, clean-cut sinuses, but these more distal sinuses are relatively shallow, so that one can with but scant propriety speak of the terminal lobe as a definite morphological structure; 
there is no incision on either margin of the lateral lobes and hence no rounded lobe corresponding with what I have called the "secondary lobe" in B.bp. heteris, though there may be a slight expansion of leaf-tissue in that region, especially in strongly developed specimens, which no doubt corresponds to the secondary lobe (fig. 9). All the lateral lobes tend to be more or less slender, elongated, and acute. In the more robust specimens there is apt to be some secondary lobation, but in all cases these secondary lobes are also attenuate. A secondary spur directed outward

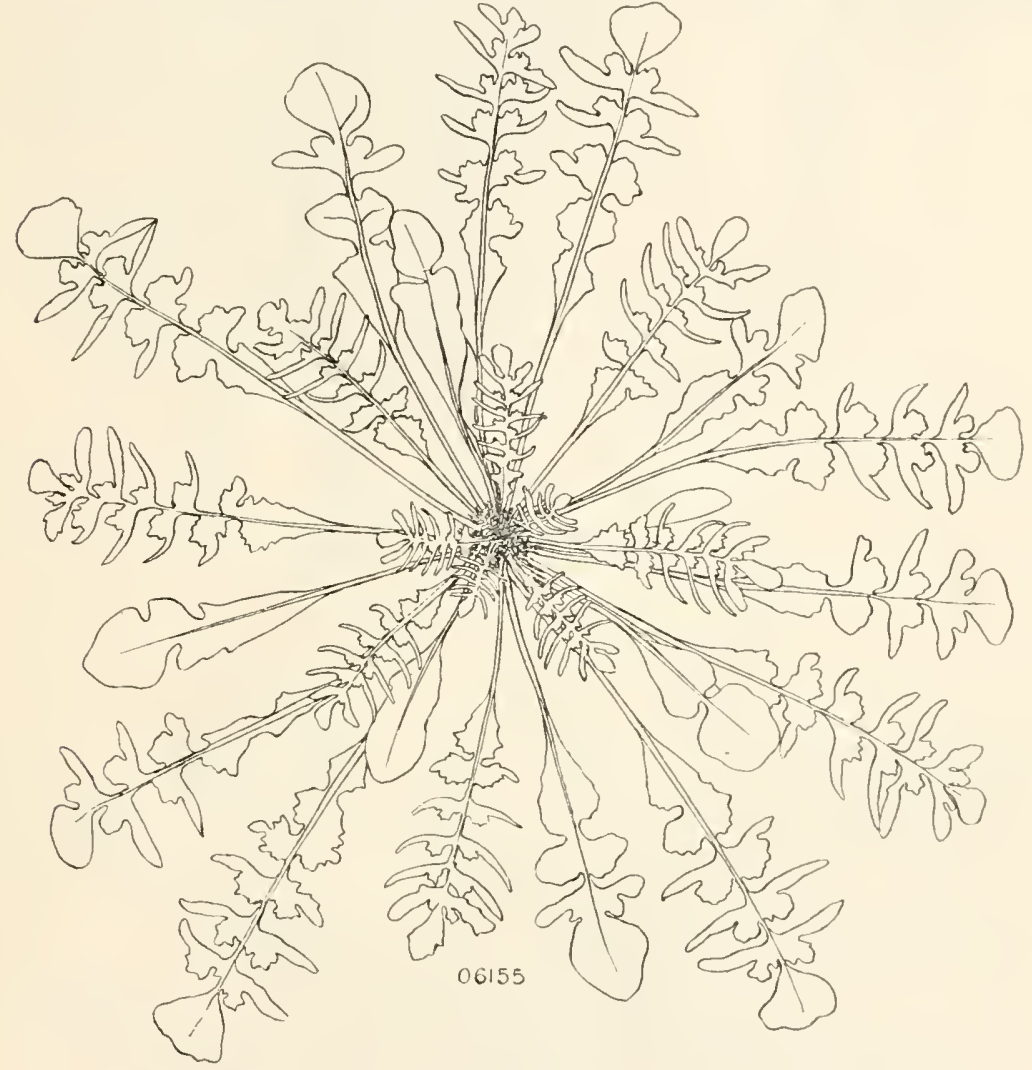

Fig. R.-Bursa bursa-pastoris heteris (?) grown from seed received from Tucson, Arizona.

and proximad from near the base of the lateral lobes is often noted in well-developed plants belonging to this biotype (fig. 10). In many instances the long, slender lobes are somewhat recurved at the tips, but in other eases all lobes are practically straight. Under this type may belong Almquist's C. bp. pedemontana (his fig. 17), leontodon (fig. 31), dentata (fig. 35), lacerata (fig. 37), bergiana (fig. 38), la.ra (fig. 39), querceti (fig. 40), ramselensis (fig. 41), and linearis (fig. 58). 
Fig. 11.
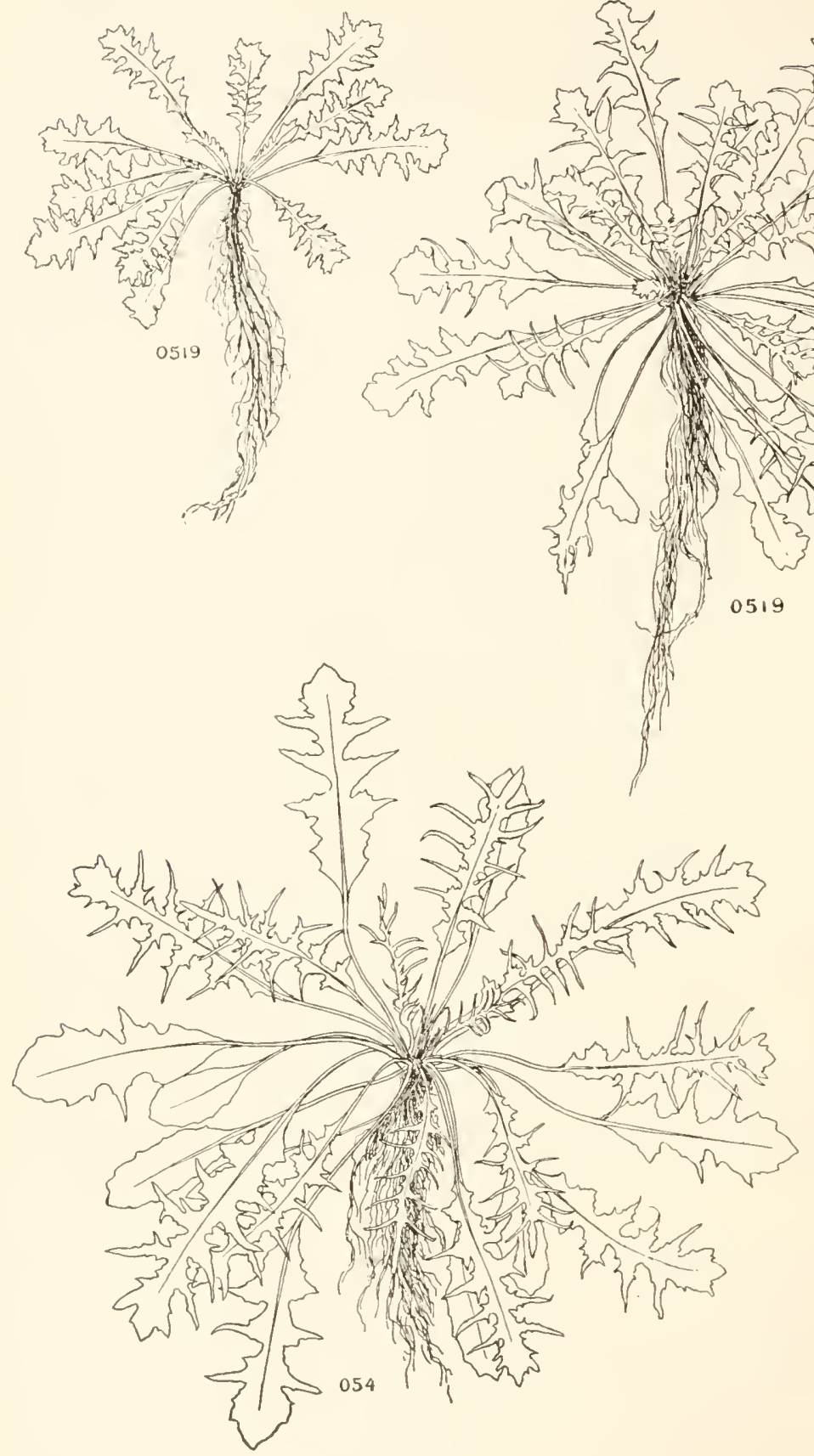

Fig. 10 .

FIG. 9.-Bursa bursa-pastoris tenuis from my first pure culture of this biotype. FIG. 10.-Bursa bursa-pastoris tenuis from a hybrid progeny.

Fig. 11.-Bursa bursa-pastoris tenuis. A stunted sib of plant shown in fig.9. 


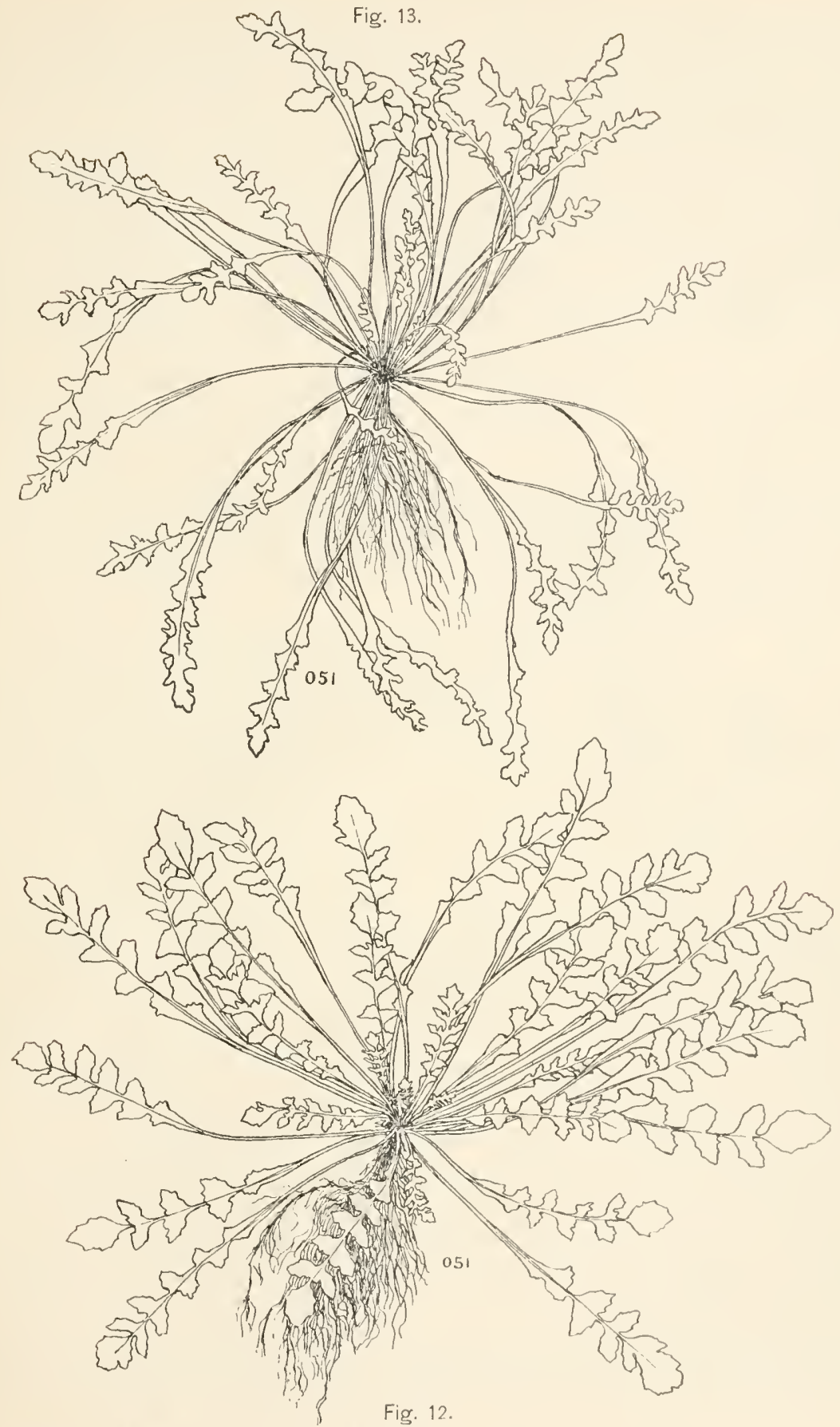

FIG. 12.-Bursa bursa-pastoris rhomboidea. Grown in best light-relations pos. sible in sky-lighted room. Characteristic incisions not well developed.

FIG. 13.-Bursa Bursa-pastoris rhomboidea modified by growing in dim light. 
The two specimens (040.19 and 040.26) which form the basis of all $\mathrm{my}$ pure-bred cultures of this biotype, germinated in vessels of earth orer which were unpacked Bursa seed which had been sent by Prof. H. F. Roberts, of Manhattan, Kansas, and as the soil of one of these vessels had been sterilized it seems almost eertain that these plants came from seed sent from Kansas. Seeds of these two plants were sown September 22, 1905, and January 31, 1906, and together gave a progeny of 361, all but 4 of which were referable withont question to the type of the parents. These 4 specimens in family 0519 which were not quite in agreement with the rest had the lobes comparatively broad and short, though still strongly aente, and the margins were slightly crenulate or denticulate (fig. 11). As these specimens were somewhat stunted, it is thought that their differences may have been due to the causes producing the stunting, but this point has not yet been tested.

Besides these two pure-bred families of $B$. bp. temuis coming prestmably from Manhattan, Kansas, this form appeared as a component of a hybrid family received from Edgewood, New Carlisle, Ohio, and also as a member of a hybrid family from Chieago, Illinois. These hybrid families will be discussed later.

\section{Bursa bursa-pastoris rhomboidea n. sp. element.}

This, like $B$. bp. heteris, has the leaves divided to the midrib and possesses a similar, more or less rhombic terminal lobe, set off by deep sinuses from the nearest lateral lobes. Each lateral lobe of the climax-leares usually shows a prominent ineision on its distal margin, by which a lobe is formed next to the rachis, corresponding to the secondary lobe of $B . b p$. hetcris, and in well-developed specimens there are usually one or two similar incisions on the proximal margin. All lobes formed by these incisions are usually obtuse or broadly angular. The terminal portion of the lateral lobes has in the best-marked examples a nearly rhombic form, which siggested the name (see figs. 18 and 20, and plates 2 and 4 ). When grown under unfavorable conditions the characteristic incisions may be lost, the prominent incisions setting off the rounded seeondary lobe being the most persistent. Almquist's C. bp. subalpina (his fig. 46), densa (fig. 51), polyedra (fig. 52), and perhaps two or three others may belong here.

Three pure cultures demonstrated to belong to $B$. bp. rhomboidca have been reared from specimens or seeds brought in from nature, and the same elementary species has been included as hybrids in two other original families. Complete proof of its elementary eharaeter will appear bulow in the section on hybrids.

Cultures derived from two plants growing side by side near the Prooklyn

Institute's Marine Piological Laboratory at Cold Spring Harbor, Long Island, were thotght for a time to belong to a biotype distinet from the 
more characteristic specimens of $B$. bp. rhomboidea secured in hybrid families. These two individuals were growing in a situation which received only the morning sun. The leaves were divided to the midrib, but the oblong, obtuse lateral lobes frequently had no incisions, though in some cases the middle lobes of the leaves had the more characteristic incision on the distal margin and less frequently a similar incision occurred on the proximal margin. The lobes were rather distant from each other, giving a very unique appearance to these plants. Seeds of these two supposed sibs were sown on June 6 and 14, 1905. The first of these (040.1) produced 65 offspring; which were studied in their relation to different conditions of environment in order to get a clue to the susceptibility of Bursa to immediate modification by variations in the chemical and physical conditions of the soil, differences in soil-moisture, atmospheric humidity, intensity of light, etc.

Specimens which were grown under as farorable conditions as the sky-lighted room prorided were essentially identical with the parent (fig. 12). Aside from the complete suppression of lobes and long delay of the flowering period in dim light (figs. 13 and 14), the most marked effect of environment was noted in plants kept in a nearly saturated atmosphere attained by cov-

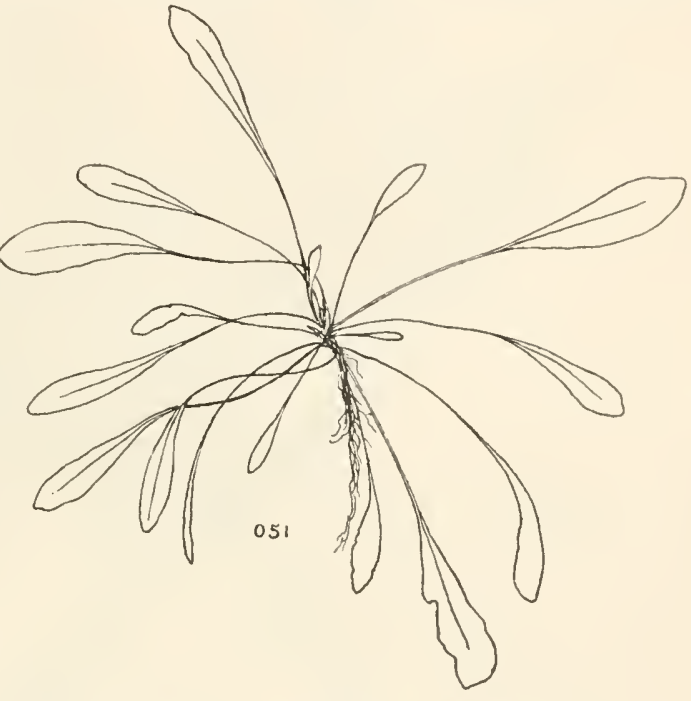

Fig. 14.-Bursa bursa-pastoris rhomboidea. A sib of plants shown in figs. 12 and 1:3, and of the same age, showing the complete suppression of lobes. This plant was grown for five months in a poorly illuminated corner of the sky-lighted room.

ering with glass jars. The leaves became long, of very thin membranons texture, crinkled and otherwise distorted, and with very shallow sinuses (fig. 15). Plants which had been grown in the dim light of the sky-lighted room of the laboratory and which showed a consequent reduction of the lobes returned to the fully lobed condition upon being remored to the betterlighted propagating-house. Seeds of one of these (051.19) were sown March 6, 1906, and produced a large progeny, only 100 of which were potted up and studied. These were uniform throughout, but as they were stunted by unfavorable conditions in the propagating-house it ras impossible to determine with eertainty their relation to other cultures which had been grown under better conditions. 
The second of trro original plants of $B . b p$. rhomboidca (040.8) produced 330 offspring, all but 5 of which possessed characters recognized as properly belonging to the parental type, namely, distinct obtuse lateral lobes, usually with an incision on the distal margin and sometimes with a similar incision on the proximal margin (fig. 16). The 5 individuals which were aberrant from the type of the other 325 had the essential characters of $B$. bp. heteris. As will be seen later, heteris is dominant over rhomboided, and the simplest explanation of the presence of these 5 specimens of heteris in

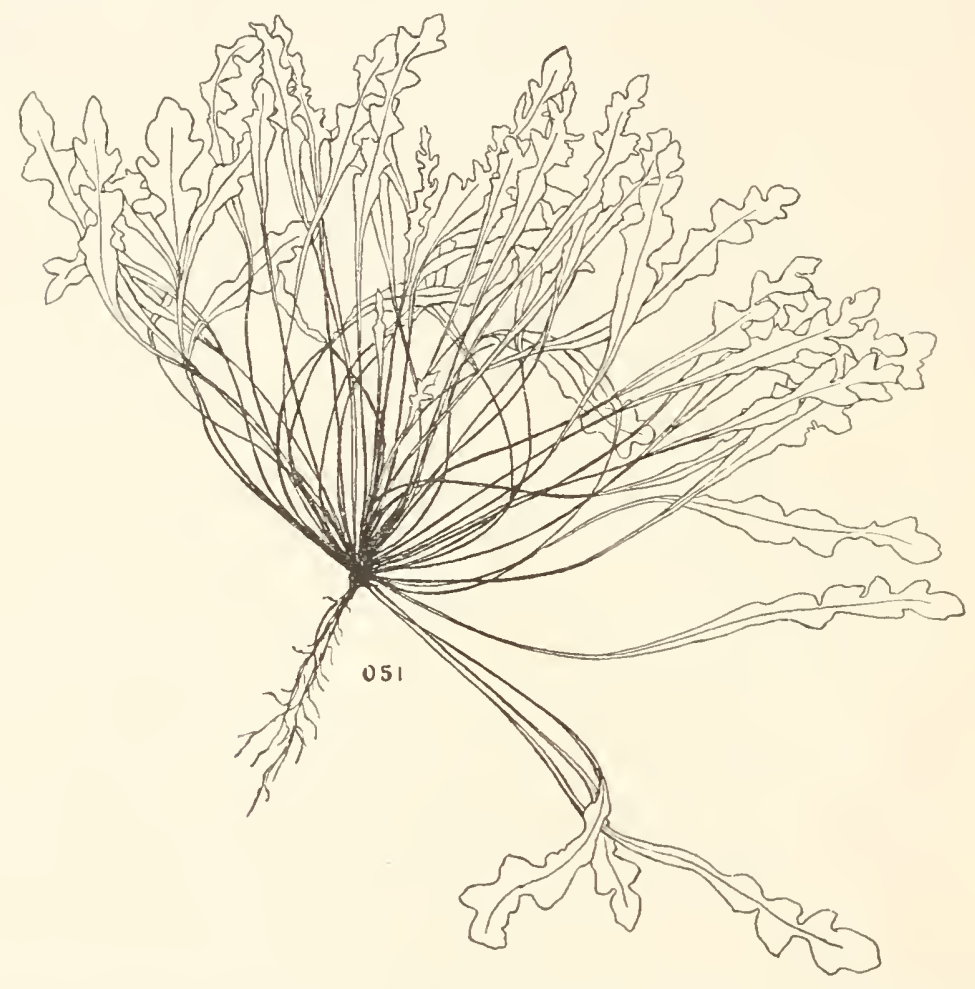

FIG. 15.-Bursa bursa-pastoris rhomboidea. Grown in nearly saturated air and moderate illumination. Leaves very thin and translucent.

a family of rhomboidea is the supposition that they represent chance crosses between these two biotypes.

Another culture which was not at first thought to be referable to $B$. b rhomboidea was raised from seeds of a plant (050.82) growing in a dooryard near Cold Spring Harbor, Long Istand. The parent had rather small leaves with crowded lobes and coriaceous texture. The lobes had a strongly marked distal incision and rarely a slight proximal incision (fig. 17). This plant was remored to the greenhouse April 18. 1906, and the flowers were 
guarded against cross-pollination. The 150 offspring (06190) were somewhat diseased and stunted for a time, but subsequently developed 11ndoubted characters of $B$. bp. rhomboidea (fig. 18).

Bursa bursa-pastoris simplex n. sp. element.

This biotype is like $B$. bp. tenuis in that the sinuses nerer reach the midrib, but it differs in having mostly simple rounded or triangular acutish lobes, not attenuate. No incisions are seen in the lobes and there is no secondary lobing, even in the most vigorous specimens, except some slight

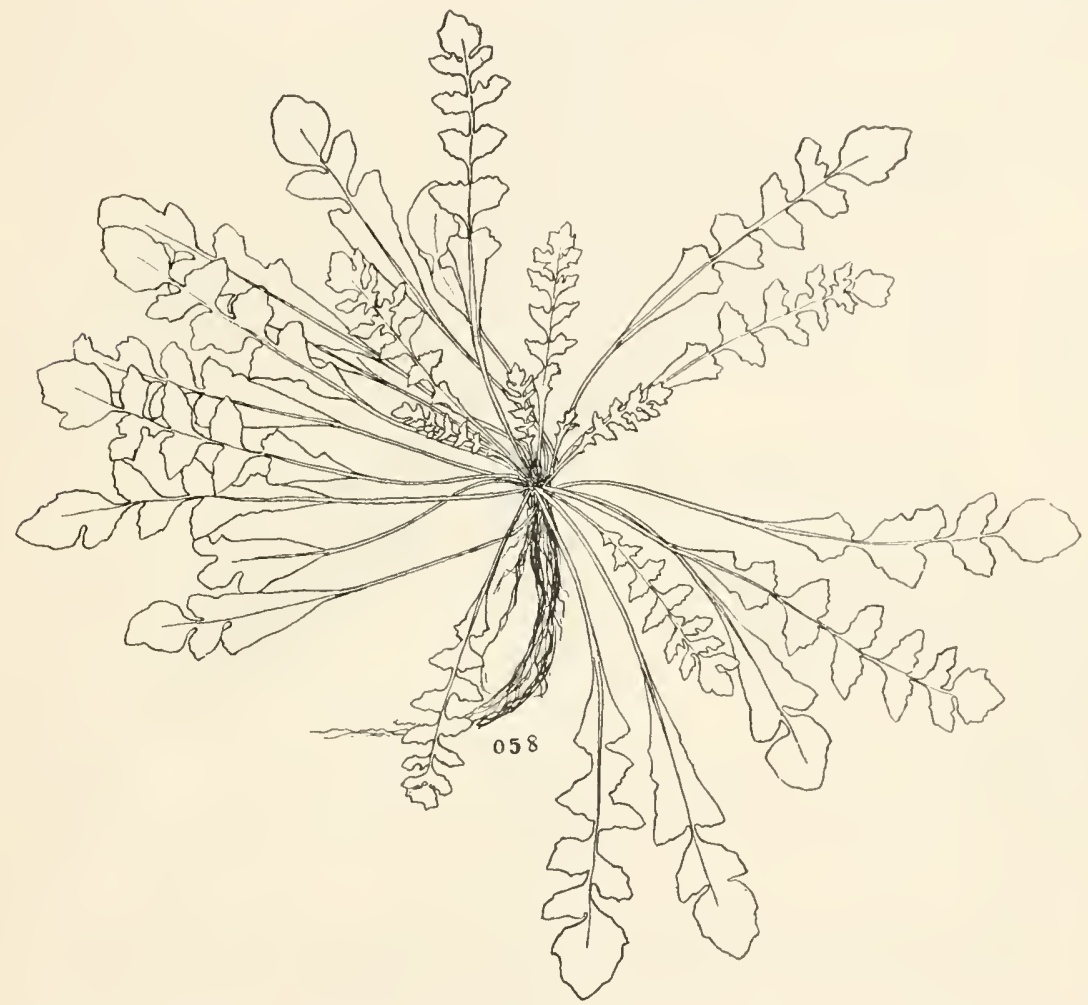

FIG. 16.-Bursa bursa-pastoris rhomboidea from the second original family.

denticulation (fig. 19). I am in doubt as to whether any of Almquist's named forms can be referred to this biotype, though it is possible that his C. bp. hauniensis (his figs. 33 and 34 ) is identical with my B. bp. simplex. His C. bp. gallica (fig. 62) and several others in which lobes are almost or quite absent might be placed here, but in most cases his figures show eridence that the plants had lost their characteristic lobing throngh some nnfavorable cultural conditions, and if this was the cause of the reduction of the lobes, such specimens might belong to any other biotype. 
Two original enltures of $B$. bp. simplex were grown from seeds collected at Edgewood, New Carlisle, Ohio, by J. Marion Shull, June 2 to 17, 1905. In the parents of both these cultures (040.5 and 040.6) the lobes were undivided and not elongated, though they were somewhat triangularacutish. One of these (040.6) had a more tapering apex than the other and its lateral lobes were more divaricate and more acute, but their progenies were generally indistinguishable from each other. Only one specimen among the offspring of 040.6 had the lons, tapering apex of the parent, this fact apparently showing the difference between the two parents in this regard to be a simple fluctuation.

These two lots of seed were sown June 26, 1905. The first (040.5) produced 170 plants which, except for some slight fluctuations, were evidently of a single type. A second generation of 44 plants raised from the seeds of an average specimen (055.24) kept strictly to the same type. The other original culture (056) consisted of 425 specimens, all but 5 of

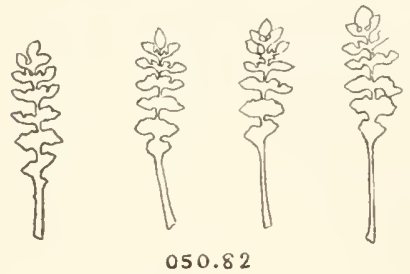

FiG. 17.-Bursa bursa-pastoris rhomboidea. Climax-leaves of a plant growing in a dooryard near Cold Spring Harbor, Long Island. which were typical $B$. bp. simplex. The 5 aberrant specimens belonged to $B$. bp. rhomboidea (fig. 20), whose presence in this family was assumed to be due to chance crosses in nature. This assumption was tested by rearing a family from seeds of one of these (056.130). Its hybrid character was fully demonstrated, and the results are given in detail in the section devoted to hybrids (p.42).

Some fluctuation was observed among the specimens of $B$. bp. simplex. A very few somewhat stunted specimens had the leares smaller than normal, somewhat shining, and with the lobes more crowded. One of these (055.103) was tested. Fully guarded seeds were sown Ju1y 18 , 1906 , and produced 48 specimens, all but one of which were typical $B . b p$. simplex, the one slightly aberrant specimen having a more coriaceous texture and slightly more distant lobes than the others. It is probable that this also represents a mere fluctuation. Other variations among the members of the original families have not been fully tested as yet. Thus in one the leaves were broader than in the usual form, in another the sinuses were deep and the lobes rather long, strongly divaricate, and acutish. There can be little doubt that these are fluctuations which would be but slightly if at all apparent in their offspring. 


\section{A VARIABLE SERIES OF DOUBTFUL SIGNIFICANCE.}

Besides the four forms named and described above, which hare been shown to breed true to type with only slight fluctuations, most of my attention has been given to a series of eultures whose behavior has becn up to the present time quite baffling. Some time I hope to understand this group better, and I shall then have more to say about it, but its behavior is in such striking contrast to that of the biotypes already described that it seems only fair to give a short epitome of my results as they now appear.

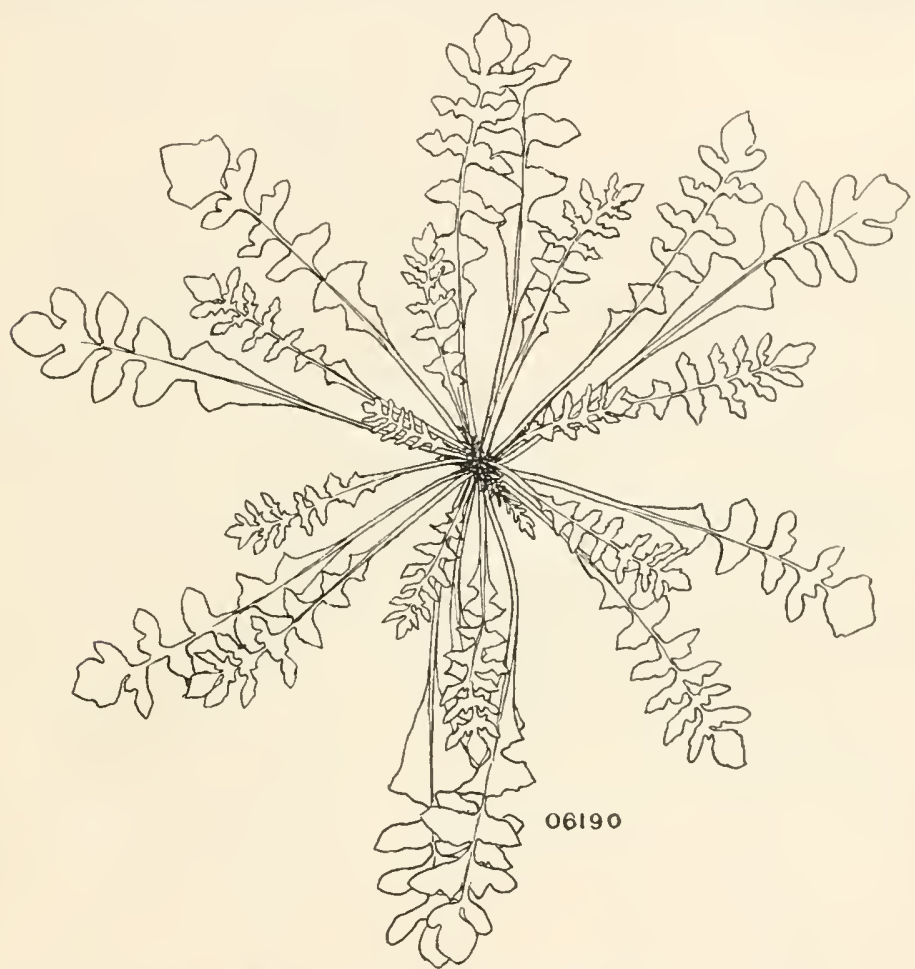

FIG. 18.-Bursa bursa-pastoris rhomboidea grown from guarded seed of the plant shown in fig. 17 .

Two specimens (040.2 and 040.7) were taken into the sky-lighted room from different habitats near Cold Spring Harbor, Long Island, in the very beginning of these cultures, April 15 to 20, 1905, and allowed to ripen seed. The aspect of these two speeimens was very diverse. One (040.2) was robust and had rather firm, thickish leaves with 4 or 5 pairs of oblong, obtuse, wavy lobes, while the other (040.7) had a small rosette with thin, flat leaves and few triangular lobes. Notwithstanding these differences, the offspring of the two plants, as followed in numerous cultures through several successive generations, were indistinguishable from each other. 
The seed of the first of these (040.2) was sown June 6, 1905, and gave a progeny of 262 individuals not quite equal to each other and not then well understood, because these were my first cultures of Bursa. These plants were nearly uniform in their membranous texture, smooth surface, and rather light-green color, but in the character of the lobation considerable differences were noted. The most common form, and that considered in consequence to be typical, had only obtuse lobes resembling $B$. bp. simplex. Besides these obtuse-lobed specimens there were about 4 which had the lobes decidedly acute or elongated.

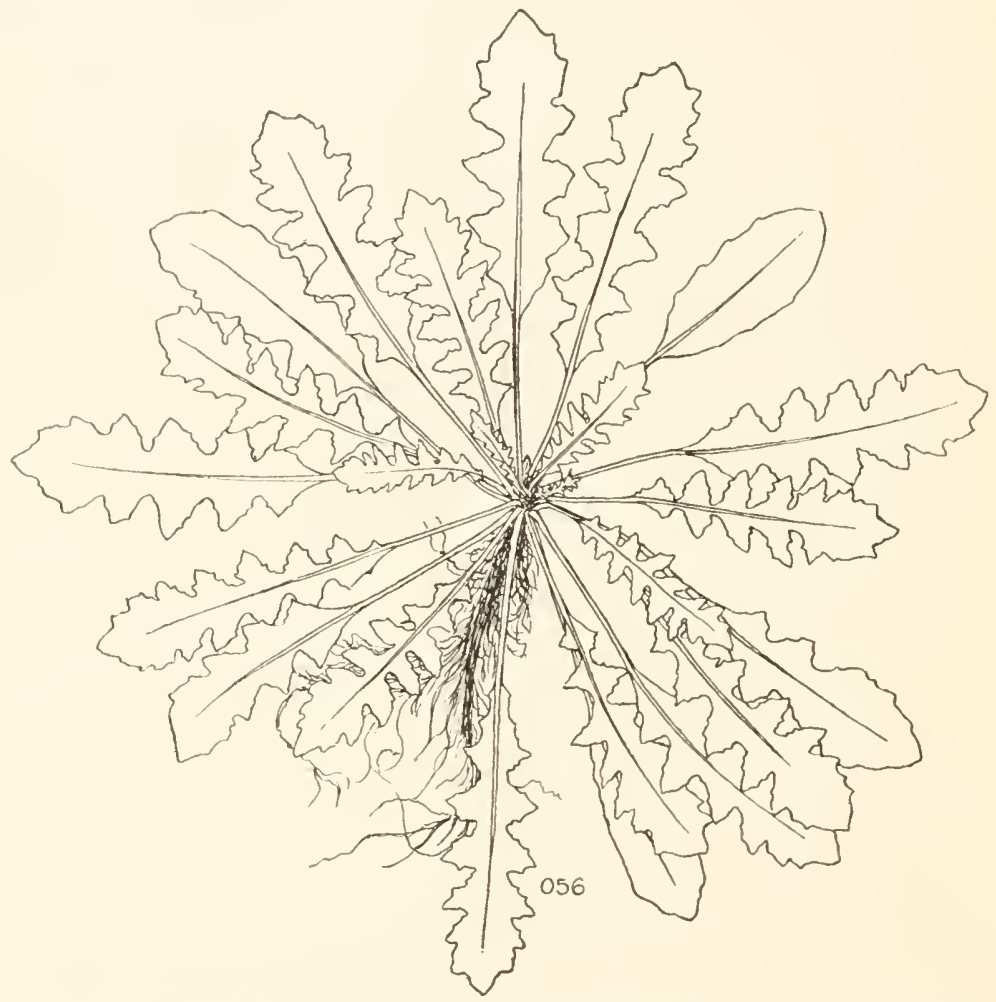

FIG. 19.-Bursa bursa-pastoris simplex grown from seeds received from Edgewood, New Carlisle, Ohio.

Seeds of the other original specimen (040.7) were sown May 8, 1905, and produced 70 offspring, about 40 of which were obtuse-lobed. The rust varied through acute lobes not elongated, somewhat elongated acute lobes with slight serration on both margins to forms with well-marked attenuation of the lobes similar to those of B. bp. tenuis deseribed above. Most of my cultures of Bursa have been made to determine the status of the variations in the offspring of these two plants and their suceeeding generations.

Before taking up the discussion of particular cultures, it may be stated that these variations from obtuse lobes and shallow sinuses to more or less 
attenuate lobes, and many other variations, have behaved in many respects as if they were the normal fluctuations of a single biotype. While in certain cases there appeared to be a marked capacity of one or the other extreme to transmit its character to its offspring, the ustal result of breecling any one of these variations was a progeny giving again the whole range of fluctuation, or a considerable portion of it. Time and again forms were picked out so different from their sibs that they were thought to represent distinct elementary species, but breeding-tests showed that their offspring return completely to the usual condition of the other related families.

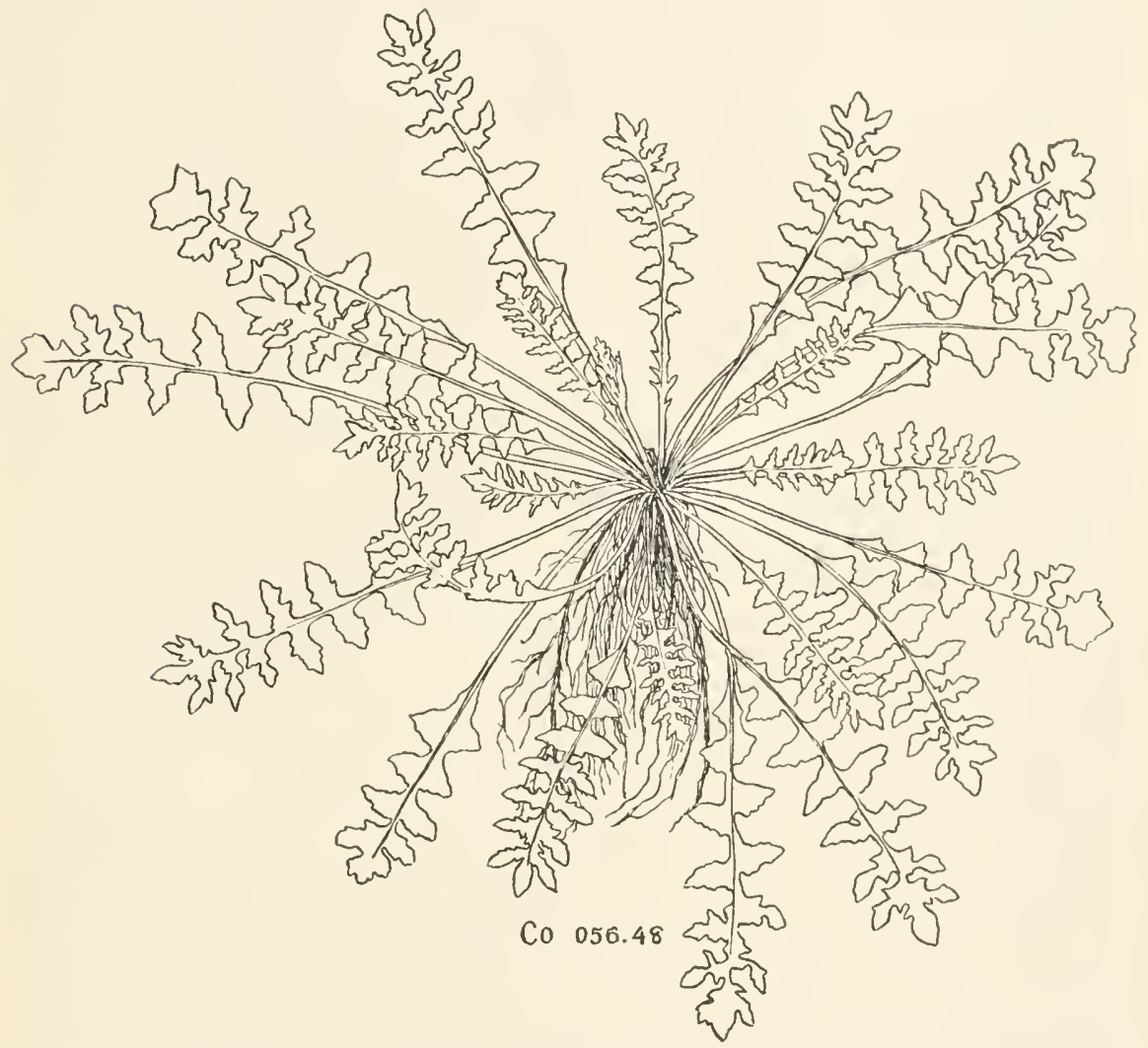

FiG. 20.-Bursa bursa-pastoris rhomboidea from a family of B. bp. simplex. A half sib of the plant shown in fig. 10.

Over 100 pedigreed families, including more than 15,500 individuals derived from the two plants $(040.2$ and 040.7$)$, have now been studied, and of these families considerably more than half ranged between forms with very obtuse lobes and others having greater or less attenuation of the lobes, the extreme developments in the latter direction being scarcely distinguishable from pure-bred $B$. op. tenuis; 17 of the remaining families, in which no attenuate-lobed element was noted, had been injured by too long crowding 
in the seed-pans. This cause, with the ravages of thrips, in many cases so affected the development of the plants during several months of the summer of 1906 that it was often very doubtful just how much dependence was to be placed upon the observed results. Howerer, enough of the families developed healthily to render it not improbable that all degrees of variation between completely unlobed individuals and those with highly dereloped attenuate lobes may be normal fluctuations of a single unit-form.

Of the healthy cultures several may be taken as examples of the peculiar beharior of these plants. Several noteworthy instances were presented in which the conditions of the parent were transmitted to the offspring with only a narrow range of fluctuation. Thus, in the second of the two original families from which all of these variable cultures sprang, there were among 70 specimens 13 which had attenuate lobes. Seeds of 1 of these attenuate-lobed specimens (057.20) were sown October 9, 1905, and produced 681 plants, all but 1 of which showed almost uniformly strong development of attenuate lobes. One specimen was entirely free from lobation of any kind, but it produced no good seeds and therefore it was impossible to test the signifieance of this unlobed condition. One of the attenuate-lobed plants of this family (0520.196) produced 35 more or less attenuate-lobed offspring, and some which were unlobed, but the latter were so crowded in the seed-pan that stunting might be considered the cause of the suppression of attenuate lobes. Progenies of 4 of these stunted individuals (05196.137, 05196.136, 05196.137, and 05196.147) have since been examined. Two of these 4 families (06136 and 06137) had the wide range of variation usually found in the related families, and the other 2 (0613t and 061+7) contained unlobed or slightly obtuse-lobed specimens. Since these last 2 families were badly crowded, little dependence is to be placed upon this behavior.

Leaving out of account all the families which may have been injured by crowding or otherwise, in 3 other instances the offspring were uniform and in fair agreement with the parent. All 3 of these belonged to the first of the 2 original families. Seeds of a well-dereloped obtuse-lobed individual (052.24) sown Janttary 16, 1906, produced 100 specimens of uniform aspeet, with only well-developed obtuse lobes. An obtuselobed sib of the last (052.179) was the parent of 130 plants of the same uniform character, having well-marked sinuses, but acutish, not elongated lobes. One of these $(05179.170)$ produced about 30 obtuse-lobed offspring, but thrips injured them so much that their characterization was unsafe. Another obtuse-lobed plant (052.193), a sib of 052.24 and 052.179 just described, gave a progeny of 125 plants of very uniform aspect throughout and always with well-developed sinuses and obtuse lobes.

In a11 these exceptional cases there scems to be a consistent behavior in that the parental character dominates the entire progeny, but in each 
such case there were sibs having the same external characteristics, which behaved in an altogether different way when bred. Thus, a sib (057.25) of the attenuate-lobed specimen (057.20) noted above as producing offspring uniformly of the parental type, was, like that plant, attenuate-lobed, but its offspring, instead of agreeing with the parent, consisted of 1 plant entirely without lobation, 168 with obtuse lobes, 186 with some of the lobes slightly elongated, and 415 with attenuate lobes. This result looks very much like a case of Mendelian inheritance if we assume that the parent was a DR, but it is not at all in accord with such an assumption that in like manner obtuse-lobed sibs of the plants whose entire progenies were characteristically obtuse-lobed as described above have produced offspring ranging from the totally unlobed condition to the well-marked attenuatelobed extreme. For example, one of these obtuse-lobed plants (052.182) whose seeds were sown February 12, 1906, produced a family of 471, of which 337 were observed to have the following composition : 11 were wholly unlobed, 43 were unlobed in the distal half of the leaf, but had small triangrilar lobes in the proximal half, 89 were obtuse-1obed throughout, 102 had some lobes slightly elongated, and 192 had some lobes strongly attenuate.

Besides a few families that were left crowded too long in the seed-pans to allow of a satisfactory estimation of the foliar characteristics, 15 additional families were reared from parents which had attenuate lobes and all gave uniformly the same result, namely, progenies showing the complete range of variation from a wholly unlobed condition to the attenuate condition of the parent; 17 families from obtuse-lobed parents had the same composition, as did also 7 families from unlobed parents.

Besides these variations, which can be easily arranged in a simple linear series, there were noted several variations of so definite a character as to lead to the attempt to segregate them as distinct forms. Thus one form which appeared in several families (e. $g ., 0527,05182$, ctc.) was characterized by a distal unlobed half of the leaf and a proximal half with triangular lobes. Another group of specimens (0623) of strikingly uniform appearance had very robust rosettes with leaves broad, obtuse, and entirely unlobed except for the presence, occasionally, of a few shallow triangular lobes at the very base.

If further breeding should confirm the conclusion that these families belong to a single biotype, including within its normal range of fluctuation individuals with obtuse lobes or no lobes at all and others with strongly marked attenuate lobes, this form would be indistinguishable in some of its phases from $B$. $b p$. simplex, and in other phases it would closely resemble $B$. bp. tenuis. Only breeding-tests could safely distinguish these several types, and so long as the practical taxonomist's work consists entirely in the classification of individuals as they occur in nature, he would be justified no doubt in refusing to recognize such elementary species as 
of working value, but in the taxonomy of the future they must be reckoned with, because they are the real natural entities with which all students of biology must deal. The old name and the old delimitation of Bursa bursapastoris may remain as the only thing practicable for the amateur collector of plants, but the morphologist, the physiologist, the ecologist, and the evolutionist must be more discriminating. It appears to me that systematic botany stands at the parting of the ways. Either it is to be left stranded as a caterer to the amateur or it must adopt the cultural method in lieu of the herbarium method, which has until recently held almost supreme sway among systematists.

I am not fully convinced, however, that the variable families here deseribed belong to a single biotype. The fact that both extremes have in certain cases bred true leads to the question whether there is not some way of accounting for the anomalous behavior of the other families on the ground of hybridization. I believe that such an explanation can be found on the principle of latent characters which I have recently discussed elsewhere (Shu11, 1908). At certain times the cultures have become very unhealthy on account of the attacks of thrips and other causes, such as overheating during the summer, crowding in the seed-pans, etc., and the specimens weakened in this way may very well have failed to show their distinctive characters, owing to what I have called "latency due to fluctuation," thus destroying completely the ratios by which hybridization phenomena would have been rendered evident.

Whether these plants belong to a single biotype of wide variability or to a hybrid group whose nature has been obscured by latency will have to await further cultures. Lotsy (1906) also found that while some forms bred true with very slight fluctuation, the variability of others was considerable. If hybridization with latency accounts for the behavior of my variable families it may also perhaps account for a similar condition in Lotsy's material. Whatever the situation may be in these highly variable cultures, it is perfectly certain that the true-breeding forms are distinct and elementary, as will be more fully demonstrated in the next section.

\section{HYBRIDS BETWEEN BIOTYPES OF BURSA BURSA-PASTORIS.}

Although artificially produced hybrids between the several biotypes above described have been studied as yet only in the first generation, except in the case of one $F_{2}$ family to be described hereafter (see page 42), I have had under observation 31 hybrid families belonging to a second or later generations. My good fortune in being able to report on these secondgeneration hybrids is clue to the facts that two of the original families were the offspring of hybrid individuals growing in nature and several other individuals in the original cultures were obviously produced by pollination from another biotype. The first of these natural hybrids (040.4) was col- 
lected by J. Marion Shul1 at Edgewood, New Carlisle, Ohio, May 26-28, 1905. This specimen was robust and the lobes of the leaf were lanceolate, with prominent dentations on both proximal and distal margins.

The seeds were sown June 26, 1905, and produced a progeny of 284 individuals, among which there was such a varicty of form and aspect as to make them at that time entirely inexplieable, because none of the unitdifferences between the forms was then known and there was no possibility of discriminating between minor fluctuations and the distinguishing characteristics of the several elementary species. Efforts were made to arrange these plants into groups that wonld be strietly homogeneous, and in this way no fewer than 25 eategories were established. Even these were not sufficient, and finally a considerable number of individuals that could not be classed with any of these were preserved in order that they might be studied at any subsequent time at which it should be desired to work out their variations and their relationship to each other and to the rest of the family.

Fortunately the distinguishing marks of each of these several groups had been noted with suffieient thoroughness that later, when the differentiating eharacteristics of the sereral elementary species were better understood, it was possible to go back and reclassify the several eategories in such a manner as to determine approximately the proportions in which the different elementary species were present. The maze of different forms had proved so baffling at every attempt at a satisfactory classification that the result of the redistribution of the several forms in the light of knowledge subsẽquently gained occasioned much surprise. There were among the 284 individuals composing the family, $114 \mathrm{~B}$. bp. heteris, $47 \mathrm{~B} .6 p$. tenuis, $53 \mathrm{~B} . b p$. rhomboidea, $16 \mathrm{~B} . b p$. simplex, and 54 which, because of certain deficieneies in the original notes, could not be reclassified with certainty, but nearly all of which were certainly fluctuations of $B . b p$. heteris.

It need only be assumed that of these 54 doubtful individuals 46 were B.bp. heteris, 6 B. bp. tenuis, and 2 B. bp. simplex to make the ratios of these several elementary forms agree exactly with a frequently observed Mendelian ratio, $9: 3: 3: 1$. Fortunately most of these doubtful specimens were preserved in the herbarium and were thus available for study. Of 51 thus preserved, 37 were B. bp. heteris, 9 B. bp. tenuis, 1 B. bp. rhomboidca, and 4 of doubtful affinity, these latter probably belonging to the hetcris group also, but representing cases of incomplete dominance.

The ratio $9: 3: 3: 1$ is the normal one for the second generation of typical Mendelian di-hybrids, $i . e$., hybrids between forms that differ from each other in two unit-characters. All of these different elementary species were supposed to differ from each other by single units until this family was worked out, beeause in nearly all the other hybrid families the simple ratio of 3 to 1 appeared. 
As soon as it was demonstrated that there are two unit-differences involved in these forms it was not difficult to discover in what these two units consist. One of them is the elongation of the primary lobes, the other the extension of the sinuses to the rachis and the presence of rounded secondary lobes in the distal axils of the primary lobes. If we represent the first by $A$ and its absence by $a$, and the second by $B$ and its absence by $b$, then we may distinguish the four members of the ratio as $A B: A b: a B: a b$. In the first, $A B$, is denoted the presence both of the elongated primary and the rotmded secondary lobe, the distinguishing characteristics of $B$. bp. heteris; in the next, $A b$, the primary lobes are attenuate, but the rounded secondary is not present, this being the character of $B$. bp. tenuis; in the third, $a B$, the romded secondary is present, but the primary is not elongated, giving $B$. bp. rhomboidea; while in the fourth, $a b$, both the secondary and the elongation of the primary are absent, resulting in $B$. bp. simplex.

The most remarkable corollary of this composition of these forms is the possible resolution and recomposition of the several elementary species. Thus, a cross between pure-bred $B . b p$. hetcris and pure-bred $B$. bp. simplex, or a cross between pure-bred $B$. bp. tenuis and pure-bred $B$. bp. rhomboidea should again give in the second generation all four forms in the ratio $9: 3: 3: 1$. The correctness of this prediction awaits the test of further experimentation.

Of the families representing the third generation of this hybrid 20 have now been studied, and the results have agreed well with the interpretation here given of the constitution of the original family. These 20 families are briefly described below in the order of the pedigree-numbers by which they were designated in the cultures and original notes.

054.184: This plant was described as of the same general type as $B$. bp. heteris, with long; slender primary lobes and a rounded secondary lobe in the distal axil of the primary. The secondary lobe usually bore 1 or 2 sharp, erect lobes. Seeds of this plant were sown February 23, 1906, and produced about 200 offspring. Owing to crowding of the propagating-house at that season of the year, only 100 plants were potted and kept for observation, the remainder being discarded with the seed-pan. The specimens potted up were taken from the pan in such manner as to prevent possible selection of individuals. Of 82 specimens noted, 80 were regarded as $B$. $b p$. heteris, and 2 as near $B$. bp. tenuis, but many of the specimens were stunted and it appears certain now that some specimens of $B . b p$. tenuis were looked upon as stunted $B$. $b p$. heteris, because the importance of the secondary lobe was not fully appreciated at that time. 'That the parent plant (054.184) was really a hybrid between B. bp. heteris and $B . b p$. tenuis is further indicated by the behavior of one of its offspring, which was 
saved as a seed-plant under the number 05184.145, and whose progeny is next described.

05184.145: This plant had the climax leaves of B. bp. heteris, but in the later rosette-leaves the rounded secondary was lost and the lobes then had one to several rather sharp incisions on both proximal and distal margins. Seeds of this plant were sown June 20,1906, and gave a progeny consisting of $158 \mathrm{~B}$. bp. heteris and $58 \mathrm{~B}$. bp. temus, or $2.72: 1$.

054.185: This plant was selected as nearly the equivalent of its parent (040.4). It had the lobes about evenly tapering and equally serrated on both margins. Its seeds were sown February 23, 1906, and gave a large progeny, which at one time suffered greatly from unsatisfactory conditions for growth during the summer months. Of these stunted specimens 236 were discarded as modifications of $B$. bp. tenuis, but 50 equivalent specimens transferred to larger pots showed that this determination was wholly unsatisfactory. Well-grown specimens belonging to this family were later shown to consist of $33 \mathrm{~B}$. bp. heteris, $48 \mathrm{~B}$. bp. temuis, $5 \mathrm{~B}$. bp. rhomboidea, and $1+B . b p$. simplex. It is plain that the manipulation of this family was such that nothing of value can be derived from the ratios, but it is worth noting that this plant, which was considered the equivalent of its parent, produced a family composed of the same four forms which had appeared in the parental family.

054.186: This plant had the lobes divided into rather narrow secondary lobes by deep incisions on both distal and proximal margins. It had some of the characters of both $B . b p$. heteris and of $B$. bp. rhomboided, but the resemblance to neither was very striking. The seeds were sown February 23,1906 , and produced a progeny which was judged to consist of $30 \mathrm{~B} .6 \mathrm{p}$. heteris, 22 B. bp. rhomboidea, and 45 intermediate between these two. These intermediate specimens differed from B. bp. heteris in the prominent incisions on the proximal margin of the primary lobes. As these intermediate forms pretty completely bridged the gap between the two parental biotypes, the wide departure from the ratio $1: 2: 1$ was doubtless only apparent, being due probably to the uncertainty of the judgment in separating the several classes. This shows that $B . b p$. heteris is not always completely dominant over $B . b p$. rhomboidea, and the same fact will be noted in several other cases.

054.190: This was a well-marked specimen of R. bp. rhomboidea. Its seeds were sown February 28, 1906, and gave a progeny of nearly a thonsand. Owing to the limitations of space in the propagating-house, only 123 of these plants were potted up for observation. One of these died. Of the remainder, all but 1 were $B$. bp. homboidea: 1 differed from the rest of the family in the elongation of the terminal portion of the lobes, this being recognized as the character of the heterozygons condition of the 
hybrid between $B$. bp. heteris and $B$. bp. rhomboidea. Had B. bp. heteris been a normal component of this family it would have made up a large portion of it, instead of less than 1 per cent. As none of the earlier cultures were guarded against cross-pollination, it seemed more reasonable to consider this one specimen the result of a chance cross produced by pollen from $B$. bp. heteris, which was abundantly represented in the house at the same time. To test further the character of the normal components of this family as well as of this one atypic individual, the following two cultures were made:

05190.167: A typical B. bp. rhomboidea, the type of the last family, whose seeds were sown J11ly 4, 1906, gave 29 plants, all B. bp. rhomboidea, showing the expected purity of this extracted form. The pollination of this plant had been carefully guarded.

05190.178: This was the atypic plant described under the last family but one. The pollination was guarded, and the seeds sown Ju1y 11, 1906, resulted in a progeny which contained 29 B. bp. heteris, 29 B. bp. rhomboidea, and 37 intermediate between the two. The deficiency of the intermediate or heterozygous class is again evidence of the difficulty of distinguishing between the extreme variations of these and cither pure-bred parent. The result here secured is sufficient demonstration of the simple hybrid character assumed for the parent.

054.208: This plant had the attenuate primary lobes rather distant and entirely lacked rounded secondary lobes. The sinuses of the earlier climax leaves did not nearly reach the midrib, but later leaves were cut more deeply. Seeds of this typical B. bp. tenuis were sown March 2, 1906, and gave a progeny consisting of $24 \mathrm{~B}$. bp. tenuis and $1 \mathrm{~B}$. bp. heteris. As $B$. $b p$. heteris is dominant over $B$. bp. tenuis, it seems likely that the specimen of the former biotype in this family represents another instance of chance crossing, since the pollination was unguarded, as in the other early cultu1'es. If this assumption is true, this family represents another instance in which an extracted form breeds true.

054.209: In the climax leares this plant had the typical form of $B$. bp. heteris. Later leaves of the rosette showed a nearly or quite complete si1ppression of the rounded secondary lobe. Seeds were sown March 2, 1906, but very few plants were secured. These were not well studied, but were observed to be heterogeneous, though most were related to $B$. bp. heteris, the parent form.

054.210: This plant was a marked example of $B$. bp. tenuis, remarkable for the extreme attenuation of its lobes. The seeds were sown March 2, 1906, and of the 31 plants capable of classification, 27 were B. bp. tenuis of strongly marked type, and 4 were $B$. bp. heteris. Unless these 4 were 
again the result of chance crosses, their appcaranee here seems at present inexplicable. A small number of speeimens in this family were too stunted by unfavorable conditions to render classification possible, and some of these may also have been $B$. bp. heteris, but it is probable that most of these stunted specimens were $B$. bp. temis.

054.3: The lobes of this plant were attenuate and rather distant, the terminal lobe spatulate or obovate, obtusish, dentate. Seeds of this B. bp. temuis were sown Mareh 3, 1906, and the 18 offspring observed were doubtless all $B$. bp.tenuis, thongh there was considerable variation in the degree of lobation, the least-lobed being stunted and not greatly different from B. bp. simplex.

054.4: This was a robust speeimen which had the earlier elimax-leares similar to $B$. bp. heteris and in the later rosette-leaves had long, attenuate, acute lobes, deeply ent on both margins. The seeds of this plant were sown March 3, 1906, and none of the speeimens were potted, but 47 were cut from the seed-pan. It was found that elassification of Bursa from the seed-pan is very diffieult and uneertain -in some cases quite impossibleowing to crowding and consequent stunting and suppression of eharaeters. The speeimens of this family removed from the seed-pan were elassified thus: $30 \mathrm{~B}$. bp. heteris, $6 \mathrm{~B}$. bp. temis, $4 \mathrm{~B}$. bp. rhomboidea, and 7 intermediate between $B$. bp. heteris, and $B$. bp. rhomboidea. It is obvious that this was a eomplex hybrid, and the number of specimens available was too small for the satisfactory working out of ratios. The excessive proportion of $B$. $b p$. heteris is easily explainable, because this form has its distinguishing marks less easily rendered latent by bad eultural conditions.

054.6: This plant had all the lobes simple and mostly obtuse. It should be an extraeted reessive, and therefore ineapable of producing any other than its own normal characteristies. Seeds were sown March 3, 1906. A11 of the plants produeed seemed to be $B$. bp. simplex, though only 1 was potted. 120 specimens eut from the seed-pan agreed with the eharacters of the parent.

054.7: The lobes of this plant were rather broadly compound, with all the lobes rounded, agreeing with the eharacters of $B$. bp. rhomboidea. The seeds were sown March 3, 1906. None of these were potted, but 43 specimens cut from the seed-pan were typical $B$. bp. rhomboide; some speeinens more nearly resembled $B$. bp. simplex, but opportunity was wanting to test this point, and a doubt remains whether these were true $B$. bp. simplex, or whether they were speeimens of $B$. bp. rhomboidea in which the charaeteristic incisions were wanting because of erowding in the seed-pan.

054.20: The plant to which this number was assigned had the long; clean-cut, primary lobe and well-marked rounded secondary elaracteristic 
of the best examples of B. bp. heteris. The seeds were sown March 6, 1906, and produced a uniform progeny of $B$. bp. hetcris, some fluctuation being noted in the denticulation of the primary lobe. None of these were potted, but 103 specimens were cut from the seed-pan, all being of the same type as the parent.

054.21: This was a speeimen of $B . b p$. rhomboidea. The seeds were sown March 6, 1906. The plants were allowed to become too erowded in the seed-pan, and did not reach their best development. Their general aspeet was homogeneous, but examination of the lobes showed some similar to $B . b p$. simplex, others more like $B . b p$. rhomboidea. The status of the simplex-like specimens in this progeny is not known, but it seems probable that many of them were really $B . b p$. rhomboidea, in which the eharacteristic ineisions failed to develop because of the erowding of the plants.

054.25: This plant was a well-developed specimen of B. bp. temis, with occasional secondary spurs on the proximal margin of the primary lobes, but with no trace of a rounded secondary lobe in the distal axils. The seeds were sown April 18, 1906, and produced 136 young plants. When examined in Angust, after my return from 2 months' absence in California, 54 were dead. The rest were all B. bp. tenuis, or modifications of it, except 3 which were $B . b p$. heteris. Unless the latter were the result of ehanee crosses their origin is not understood.

054.26: This speeimen belonged to the most distinct type of $B . b p$. heteris. The seeds were sown April 18, 1906, and produced 173 offspring. About 20 died and the remainder belonged to B. bp. heteris, and B. bp. temuis in the ratio $119: 34$ or $3.5: 1$. Some of the specimens elassed as B. bp. heteris had the primary lobe rather strongly incised on both margins. This is a character frequently seen in robust speeimens of $B$. bp. tenuis, and it is possible that this represents a slight lack of complete dominance of $B . b p$. heteris over B. bp. temuis.

054.27: This plant had large, wide-spreading, rather thickish, stiff, strongly bipinnatifid leaves, with all the lobes tapering and aente, agreeing thuss, in an essential way, with the parent (040.4). The seeds were sown April 18, 1906, and produced about 270 offspring. These are seen now to indicate that the parent was a di-hybrid, but at the time the family was being studied the distinetness and the limitations of the several forms were not sufficiently appreeiated, and the notes made at that time were in the terms of a dominant and a recessive group, the dominant group having the sinuses extending to the rachis and the terminal lobe more evenly rounded and more enneate than in the recessive group, which had the sinuses much less deep. If these distinctions were consistently made thronghout, the resilt should be the same as that of a simple Mendelian hybrid, sinee the former 
group, as described, would include $B . b p$. heteris and $B . b p$. rhomboidea, while the latter would contain $B$. bp. tem is and $B . b p$. simplcx, and the ratio of the two groups should be $3: 1$. The observed ratio was $187: 66$ or $2.83: 1$, a fair agreement with expectation. The quantitative relations of the subgroups ean not be derived from the notes, but among:146 of the dominant group discarded at one time, 25 were considered $B$. bp. rhomboidea, and the most of the remainder were intermediate between $B$. bp. rhomboidea and $B$. bp.heteris. Aceording to theoretical considerations there shonld have been $36 \mathrm{~B}$. bp. rhomboidea in that number. I have no doubt that this discrepancy was due to the fact that too narrow a vicw was taken of the fluctuations normal to the several recognized forms, so that some which were considered intermediate between $B$. bp. heteris and $B$. bp. rhomboidea were in reality extracted $B$. $b p$. rhomboidea. This is even more certainly true with respect to $B . b p$. heteris, for in the same group of 146 dominants, only 2 or $^{\circ}$ 3 were recorded as $B$. $b p$. heteris. All those having some incisions on the proximal margin of the primary lobe were considered intermediate, but pure-bred $B . b p$. heteris has since been observed to possess these incisions frequently as a fluctuating character. No $B$. $b p$. simplex was recognized, as this elementary species would have been thrown with $B$. bp. tenuis without question on the basis of depth of simus and form of the terminal lobe, which characters alone were used in the classification.

054.28: This plant was a good specimen of B. bp. rhomboidea, having the incisions on both proximal and distal margins of the primary lobes, and all the lobes on the earlier climax leaves rounded or rhomboidal. The later rosette-leaves had some of the secondary lobes acutish, but not elongated. The seeds were sown on April 1S, 1906, and 325 plants were potted. Of 302 which were studied later, 202 were classified as $B . b p$. rhomboidea and 100 as $B$. bp. simplex. This is a rather large departure from the expected ratio of $3: 1$, but here again the excess of $B$. bp. simplex may be due to the fact that the elaracteristic marks of $B$. $b p$. rhomboidea tend to disappear under unfavorable conditions of eulture, so that some that were classed as $B$. bp. simplex may have been modified specimens of B. bp. rhomboidea.

054.29: This plant was a well-marked specimen of $B$. bp. heteris, having slight denticulations on the primary lobes. It differed from the usual habit of Bursa bursa-pastoris in having the leafy portion of the stem absent. The seeds were sown April 18, 1906, and produced orer too young plants. These were readily divisible into two gromps-B. bp. hetiris and $B$. bp. tenuis - in the ratio $319: 95$ or $3.36: 1$. A few of the specimens of this family which were classified with the dominant form differed from $B$. bp. heteris in the lack of the rounded secondary. They were like it, however, in that the sinnses extended completely to the midrib, thus 
giving more nearly the aspect of $B$. bp. heteris than of $B$. bp. tenuis. I now suspeet that these should have been classed with B. bp. tenuis.

In summing 11 these 20 hybrid families belonging to the third or later generations, it appears that 8 of the 9 possible combinations are included. Two of the seed-plants proved to be extracted $B$. bp. heteris, 4 were extracted $B$. $b p$. tenuis, 2 were extracted $B$. hp. rhomboided, 1 was extracted $B$. $b p$. simplex; 3 were again like the parent in being di-hybrids which gave rise to all 4 biotypes; 3 were $B$. bp. heteris $\times$ tenuis, 2 were B. bp. heteris $\times$ rhomboidea, 1 was $B$. bp. rhomboidea $\times$ simpler, and the characters of 2 were in doubt. These 2 doubtful families (067 and 0621) were either extracted $B$. bp. rhomboidea or hybrids between $B$. bp. rhomboidea and $B$. bp. simplex, the doubt being caused by the fact that stunted or juvenile specimens of $B$. bp. rhomboidea may be practically indistinguishable from $B$. bp. simplex. In a few families the conditions of the culture made the determination of ratios impracticable, but in the majority the approximation to Mendelian expectation is fairly close. Small numbers of $B$. bp. heteris oceurred in 3 families where large numbers would have been expected if that elementary form had been a normal component of these hybrid families, since $D . b p$. heteris dominates both $B . b p$. tem is and $B . b p$. simplex, and to a slight degree $B$. bp. rhomboidea also. The 8 specimens of $B$. bp. heteris which seemed out of place among more than 2,600 individuals included in these families-less than one-third of 1 per cent of the whole-were probably the result of chance crosses, as all of these oceurred in families whose pollination had not been guarded.

040.14: The second hybrid brought in from nature was collected by C. A. Shull, in Jackson Park, Chicago, in the summer of 1905. This plant had long, acutish lobes, serrated on both margins, and the sinuses extended - practically to the rachis. There was a very faint indication of a rounded secondary lobe in some leaves. Seeds were sown December 27, 1905, and produced 99 B. bp. heteris and 26 B. bp.temis, or in the ratio 3.8: 1. The two components of this hybrid were at first considered distinet biotypes until another family bearing the same characters as the dominant form proved in the third controlled generation to be $B$. bp. heteris. Subsequent breeding has demonstrated that these hybrids also present the characters of typical $B$. bp. heteris and typical $B$. bp. temuis when grown for several generations under favorable conditions. The peculiarities of the original plant and of the first generation under culture are thus shown to have been fluctuations of these two types. Eight families of the third generation have been studied, with the following results:

0514.128: This was a specimen of $B$. bp. temis, the recessive form, and as the pollination was carefully guarded, it should have been expected to produce nothing but the parental form anong the offspring. The seeds 
were sown June 5, 1906, and produced a uniform progeny of 145 plants, all like the parent.

0514.131: This specimen was, like the last, B. bp. tenuis. Purely fortilized seeds were sown June 6, 1906, and the 52 plants produced were all alike and like the parent.

0514.146: This was of the dominant type, $P$. bp. heteris. The pollination was probably not guarded, as no note was made regarding it. 'The seeds were sown June 20, 1906, and produced $4+4$ offspring belonging to the dominant and recessive forms in the ratio $317: 127$ or $2.5: 1$. The dominant group appeared to belong to two distinct types, with the primary lobes broader and less sharp in the one than in the other in the ratio $110: 32$ or $3.44: 1$. The significance of the ratio thus formed by this family, $9: 3: 4$, is well known, as it represents the simplest modification of the ratio for the second generation of a di-hybrid, but without further data in support of this composition for the family, it appears advisable not to consider its significance at this time.

0514.161: This was a typical specimen of the recessive form, $D$. bp . tenuis, of which the pollination was guarded. The seeds were sown July 4, 1906, and prodnced 104 specimens, all of which were like the parent.

0514.162 : This was also an extracted recessive. Guarded seeds were sown July 4, 1906, and gave a progeny of 15 specimens, all like the parent.

0514.164: A member of the dominant group, B. bp. heteris. Purely. fertilized seeds sown July 4 , 1906, produced $13+$ plants. The family was badly damaged by aphis, so that 23 were killed and abont 15 more so stunted as to make classification uncertain. The remaining 93 belonged to $B$. bp. heteris and $B$. bp. tenuis in the ratio $66: 27$ or $2.4+: 1$. In this family the dominant group was not scrutinized with sufficient care to determine whether it was heterogeneous like 0514.146 , but it was considered of a single type.

0514.165: This was a typical specimen of the dominant type, B. bp. heteris. Purely fertilized seeds were sown July 4, 1906, and produced 42 specimens of $B$. bp. heteris and 13 of $B$. bp. temis, the ratio being $3.23: 1$.

0514.182: Although the notes are defective regarding the character of this plant, the result of the breeding test leaves no doubt that it was a $1^{\circ}-$ cessive. Guarded and purely fertilized seeds were probably som in Iuly, 1906, though no record can be found to that effect. On Angust 18, 1906, the entire progeny, consisting of 158 specimens, were potted, and without exception these belonged to $B$. bp. temuis.

These 8 families belonging to the third or later hybrid generation of $P$. $b p$. heteris $\times$ temis represent the extracted recessive in 5 cases and the heterozygots form in 3 . The extracted recessives have bred truc to the 
characters of $B . b p$. tenuis without exception in 330 individuals. In the offspring of 3 heterozygous plants that have been tested, the ratios $2.44: 1$, $2.5: 1,3.23: 1$, and $3.44: 1$ have appeared, and these are no doubt as near $3: 1$ as the smallness of the families should have led us to expect.

056.130: Besides these 2 original hybrid families, it will be recalled that certain unexpected individuals of $B . b p$. rhomboidea in one of the original families of $B$. bp. simplex (056) were supposed to be due to chance crosses (see p. 26). Seeds of one of these (056.130) were sown June 6, 1906, and produced 217 B. bp. rhomboided and 72 B. bp. simplex, that is, in the ratio of $3.01: 1$, thus showing the assumption that they were of hybrid origin to be correct. (See plate 2).

In addition to the hybrids brought in from nature and their self-fertilized offspring as represented by the 31 families which are described above, 7 first-generation hybrid families between different biotypes of Bursa bursapastoris have been produced by artificial crossing. Only one $\mathrm{F}_{2}$ family has been reared from these to the present time.

0515.93: The mother of this cross was a specimen belonging to the second original family of $B$. bp. heteris (0515) described above (see p. 14). This plant was castrated and pollinated with pollen from a specimen of the first original culture of $B$. bp. tenmis (0519, see p. 22). The seeds were sown May 2, 1906, and produced 222 plants, all resembling the mother and possessing the essential features of $B$. bp. heteris.

0693.203: Self-fertilized seeds of 3 guarded specimens belonging to the last-deseribed family were sown together October 12, 1906, and gave a progeny of 111 specimens, 84 of which were typical $B$. bp. heteris and 27 B. bp. tennis, or in the ratio $3.1: 1$. (See plate 1.)

As no other artificially-produced hybrids between biotypes of $B$. bursapastoris have been studied beyond the first generation and only facts bearing upon the question of dominance can be derived from these cultures as yet, it seems best to postpone their discussion until the second generation has been studied. 'This is the more important, since the specimens chosen for crossing in a number of instances belonged to families whose relations to the 4 biotypes which have been involved in the above-described natural hybrids are still in doubt.

The occurrence of supposed hybrids among the biotypes of Bursa bursapastoris has been noted by Almquist (1907, pp. 22 and 88-89), who also refers to descriptions of similar cases by Von Borbas and by Grenier. The actual hybrict character of the plants mentioned is very doubtful, however. The assmmption of their hybridity is based wholly upon the facts of intermediacy and sterility, together with the regetative vigor and longevity which are certainly correlated with sterility. I have occasionally observed such sterile or nearly sterile plants in progenies produced from seeds fully 
guarded, so that hybridity was excluded with such care as is possible. Intermediacy in the size of flowers is not at all strange in the case clescribed by Almquist, for the culture in which the supposed hybrids occurred was that of a very large-flowered form. Intermediacy in such a ease simply means a reduction in the size of the flowers. In all of my hybrids there has been no apparent decrease in the number or viability of seeds produced, and it will be recalled that there is only one clear case of intermediacy, namely, in the hybrids between $B$. $b p$. heteris and $B$. bp. rhomboidea, in which it is due to the incomplete dominance of the former. Perhaps in other cases also dominance is not quite complete, but it is so nearly so that it is impossible to distinguish certainly between the heterozygote and one of its parents.

\section{HISTORY OF BURSA HEEGERI.}

Eleven years ago Professor Heeger found some specimens of a cruciferous plant growing in the market-place at Landau, Germany, which he could not identify. In general habit these plants resembled the almost cosmopolitan species Bursa bursa-pastoris. They differed from the latter species, however, in having the seed-capsules elliptical in longitudinal section and circular in cross-section instead of flat and triangular or obcordate, as is characteristic of Bursa bursa-pastoris.

The specinens were submitted to Solms-Laubach, who was inclined at first to refer them to the genus Camelina, which is characterized by nearly spherical capsules, and Professor Ascherson, to whom he showed them, was of the same opinion. Cultures made by Solms-Laubach from seeds secured from Professor Heeger soon indicated, however, the near relationship of the new form to Bursa bursa-pastoris, when in 1895 several apparent reversions to the capsule-form of $B$. bursa-pastoris were noted. Solnus-Laubach (1900) published an account of the new form, assigning to it the name Capsella heegeri, which becomes, according to the rule of priority, Bursa hecgeri (Solms-Laubach).

This very distinct species of Bursa has attractec considerable attention, for the reason that its occurrence as a component of the flora of a region so well known systematically has left little doubt of its very recent origin from $B$. bursa-pastoris by mutation, and it is mentionect by De Vries (1901, pp. 477-478; 1905 , pp. 582-584) as an instance of mutation in nature. Shortly after the publication of the original account, Bursa hecgeri disappeared from the type locality at Landau, owing to the destruction of its habitat by covering the nrarket-place with gravel, and it has been reported from nature only once since that time, though it has been wictely grown in botanical gardens.

The second report of the discovery of Bursa heegeri in nature was made by Laubert (1905), who found it along the Dahlem turnpike in 1905, but 
here the likelihood of a new origin is certainly exceeded by the probability that a seed was carried to this spot by some agency from a nearby culture, for it had been grown for several years at Dahlem in an unprotected bed several hundred meters from the place in which Laubert discovered it. Hus (1908) takes the alternative view, however, and considers this a case of repeated mutation.* Iaubert points out that in addition to the capsule character noted by Solms-Laubach, there are other characters of the stem and inflorescence which serve to distinguish $B$. heegeri from its supposed parent and which would suggest a more distant relationship with that form than had been supposed; but, on the other hand, he found that in both species there occur frequent instances of abnormal pistillate but sterile flowers in the lower portion of the flower-stem, and he takes this fact, together with the occurrence of capsules occasionally simulating those of $B$. bursa-pastoris, formed when B. hecgeri is attacked by Albugo and Peronospora, as additional proofs that $B$. heegeri is a derivative from $B$. bursapastoris. Of the manner of its origination from $B$. bursa-pastoris nothing is known, of course, but Potonië (1906) suggests that it is a reversion induced by some pathological conclition.

More recently Noll (1907) has investigated some plants resembling $B$. heegeri, which had already been found by Melsheimer in 1882 in hundreds in a field of Dattenberger Flur and again in 1884 on a height at Linz. Melsheimert considered these plants hybrids, but could not suggest the probable parents, while Körnicke and Wirtgen† stated that they are doubtless identical with Bursa hecgeri. Specimens of Melsheimer's plants were placed in Petry's herbarium bearing the label "Capsella bursa-pastoris forma caps. ovatis." No11 received this material from Petry, together with living specimens collected by the latter at Didenhofen, Metz, Hagendingen, and Krewznach. A careful comparison of the anatomical features of these plants with those of Bursa hecgeri and B. bursa-pastoris led Noll to the conclusion that the Melsheimer plants are not Bursa heegeri, but a sterile form of b. bursa-pastoris, to which he gives the name Capsella pseudo-heegeri. The finding of these plants in considerable numbers tends to weaken the argument that the discovery of $B$. heegeri in a region so well known proves it to be a recent mutation. Perhaps Bursa heegeri will yet be discovered in some abundance in some locality where it has hitherto escaped notice.

*I have called the attention of Dr. Hus to this matter and he concedes in a letter that my explanation of the occurrence of $B$.heegeri in nature at Dahlem is probably the correct one.

$\dagger$ Mentioned by Noll, but not verified by me. 


\section{HYBRIDS BETWEEN BURSA BURSA-PASTORIS AND BURSA HEEGERI.}

The prominent part which mutation may have taken in the production of new species makes it of great interest to know, in each case, just what will be the result when the supposed or the demonstrated mutant is selffertilized and when it is crossed with the parental form; for its behavior in these two cases is the first important factor in determining the power of the mutant to maintain itself at the time of its origin and its capacity to give rise to a successful series of genetically related individuals belonging to an independent type.

The result of self-fertilization was investigated by Solms-Laubach before the publication of his original account, and he showed that the characters that differentiate $B$. heegeri from $B$. bursa-pastoris are fully heritable in a self-fertilized line. As we have seen, the Bursas normally self-fertilize to a predominant extent, and this liabit, coupled with a vigorous constitution, would seem to constitute all the factors necessary to successful maintenance. I have now determined what will be the result of intererossing $B$. heegeri and B. bursa-pastoris.

The aspects of Bursa heegeri and B. bursa-pastoris as they appeared in my cultures were so different that at the first I was skeptical concerning the near relationship which has been assumed to exist between them. Bursa heegeri was much more vigorous than B. bursa-pastoris, and the differences observed in the inflorescence by Laubert (1905) were strikingly apparent, the pedicels of the capsules being shorter, more crowded on the rachis, and diverging from the latter at a wider angle (fig. 21). While the leaves of the rosette of Bursa hecgeri are of the heteris type, having: the primary and the rounded secondary lobes readily distinguishable, the sinus which sets off the latter is comparatively shallow, and in consequence the secondary lobe appears low and less well-marked. The primary lobe is usually broader and less strongly attenuate than in $B$. bursa-pastoris heteris. Despite these considerable differences, however, it was found that $B$. heegeri may be crossed with B. bursa-pastoris with perfect ease in either direction and without any apparent decrease in fertility, though I crossed it with B.bp. simplex, the biotype of the latter species which is most unlike B. heegeri.

The families of pure-bred $B$. hecgeri and its hybrids with $B$. bp. simplex may be briefly considered under the pedigree-numbers used during their culture :

040.9: Seeds of Bursa heegeri received through Dr. D. T. MacDougal from Professor Solms-Laubach were sown July 31, 1905, and produced 26 plants, all of which agreed with the above description, the fluctuating variations being extremely slight. The unguarded seeds of one of these 


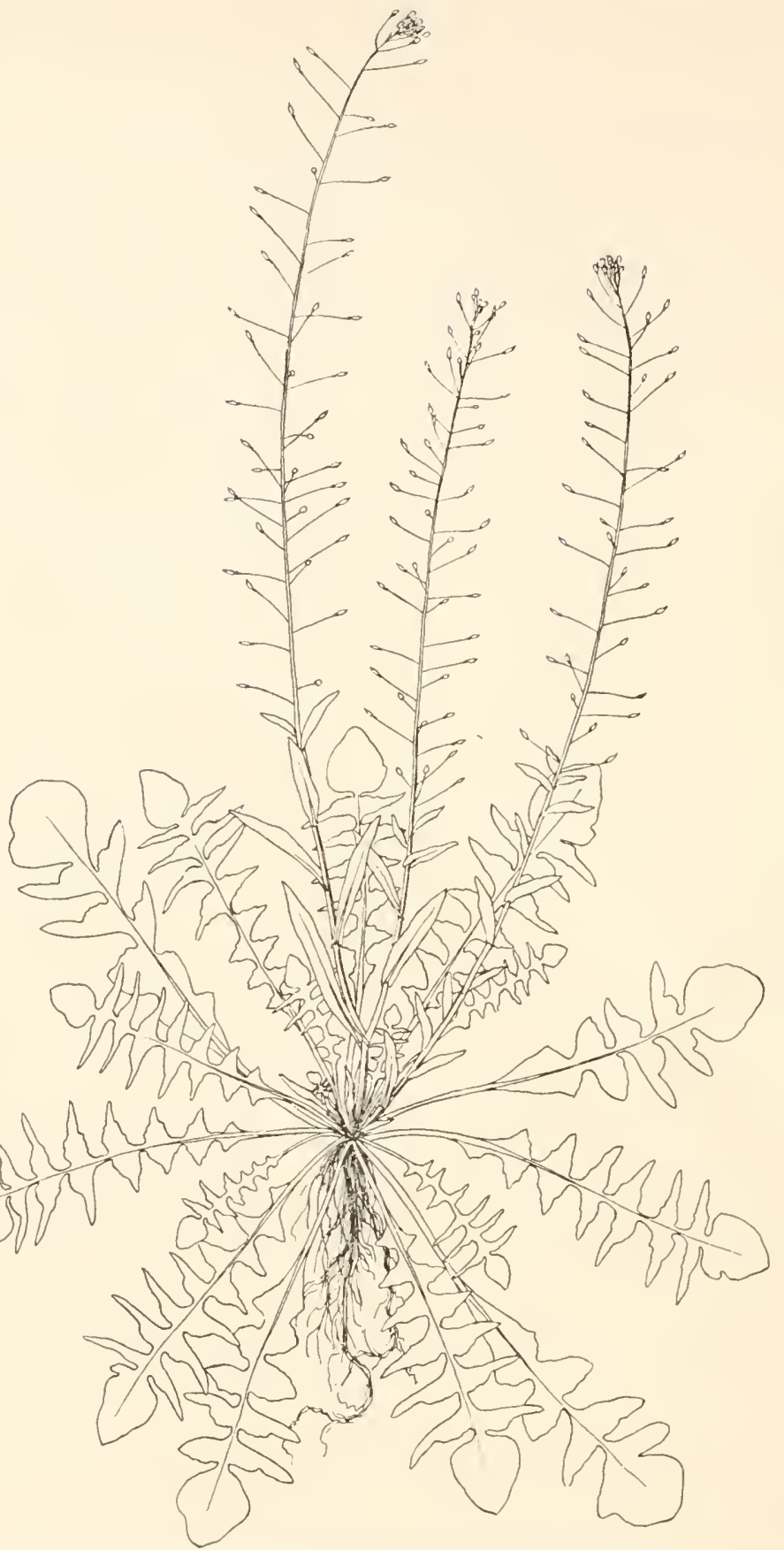

FIG. 21.-Bursa heegeri (Solms-Laubach). From photographs taken at three different stages of development. 
(059.56) were sown November 1, 1906, and produced 24 plants, all agreeing perfectly with the original type as clescribed (fig. 22). These became diseased later, however, and produced no seed.

056.88: This plant was considered a typical specimen of B. bp. simplex, though a little more vigorous and broader-leafed than usual. It was carefully castrated and pollinated with pollen from a plant belonging to my first culture of B. heegeri (059). The seeds were sown April 25, 1906, and

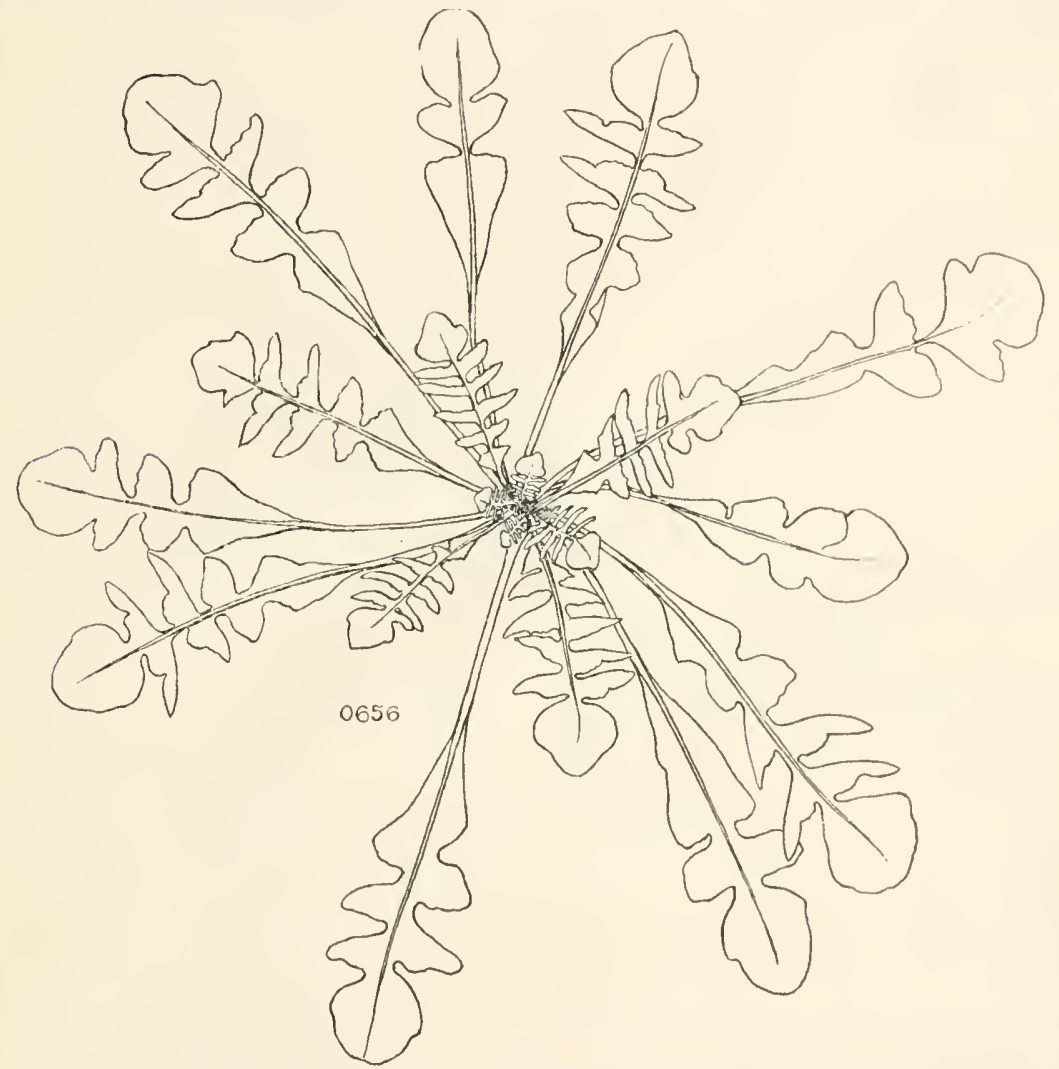

Fig. 22.-Bursa heegeri. Second controlled generation.

produced 108 offspring, all resembling $B$. heegeri more closely than $F . b p$. simplex, but they differed markedly from the former because of the imperfect dominance of the heteris characteristics. On this account these plants had some of the characteristics of $B . b p$. rhomboidea and could be properly described as intermediate between $B . b p$. rhomboidea and $B . b p$. heteris (fig. 23). A few of these died without seeding, but all that came to maturity had the triangular capsules typical of Bursa bursa-pastoris. One family was raised from unguarded seeds of one of these plants (0688.212), as described below. 
059.89 : This typical specimen of Bursa heegeri was castrated and pollinated with pollen from a specimen of $B$. bp. simplex which differed from the usual condition of that biotype in having the apex of the leaves tapering and acutish. This condition has been shown to be merely a fluctuation ( see p. 26), so that the plant used in this cross is to be considered a

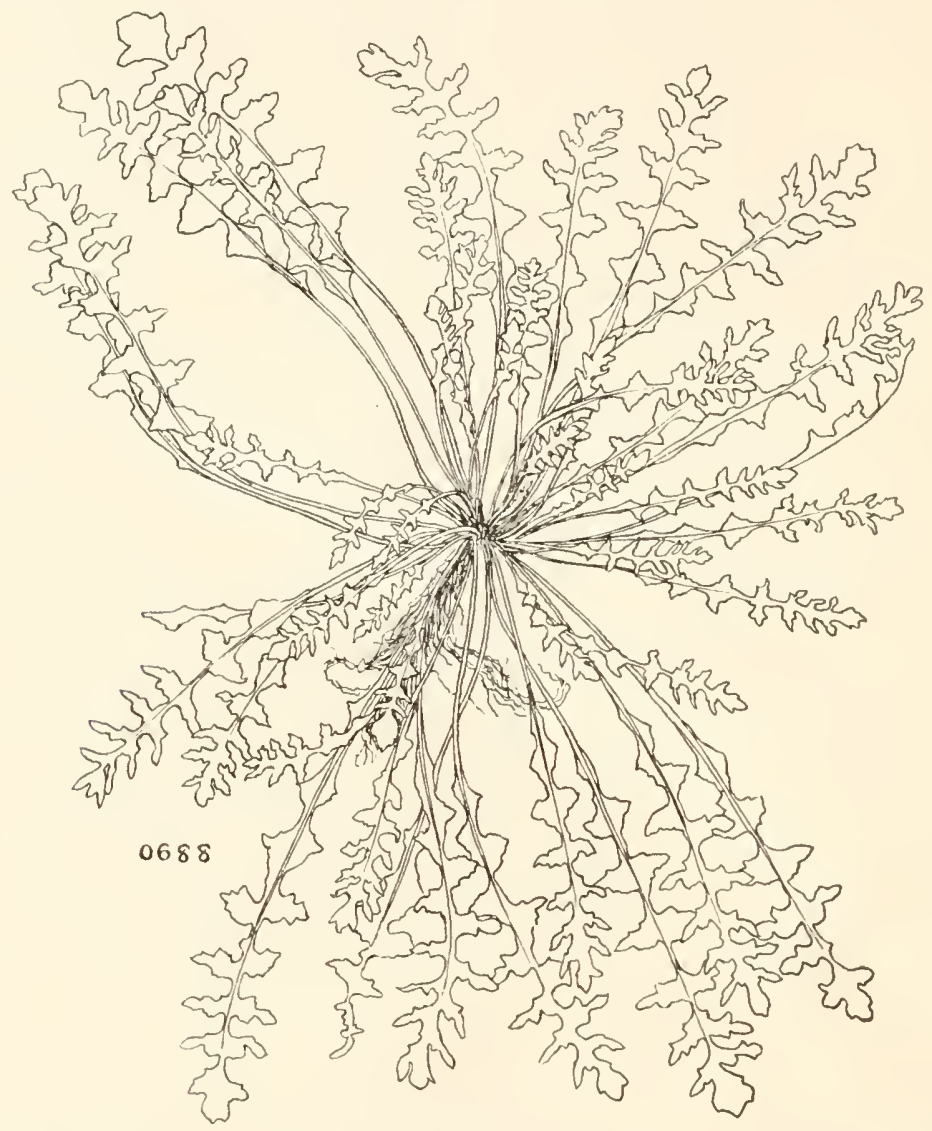

FIG. 23.-Bursa bursa-pastoris simplex $\times$ heegeri, $\mathrm{F}_{1}$. Incomplete dominance of the heteris characteristics produces a form resembling $B . b p$. rhomboidea, but mostly with longer, sharper primary lobes.

typical specimen of $B$. bp. simplex. The pollen-parent of this family was a sib of the pistil-parent of the family last described, and the two specimens of $B$. heegeri involved in these two families were likewise sibs, so that these two crosses were essentially reciprocal. The seeds were sown April 25, 1906, and produced 23 offspring, which were intermediate between $B . b p$. heteris and $B . b p$. rhomboidea as to the rosette characters, and in which they were indistinguishable from the reciprocal hybrid family, 
0688. Most of these plants produced fruit, and in all cases this was flat and triangular to obcordate, like that of $B$. bursa-pastoris. Unguarded seeds of 3 of these and guarded seeds of 1 were used for the production of second-generation families, 1 as 0689.196 and 3 collected together as 0689.197. The descriptions of the second-generation families follow:

0689.196: Seeds of this plant, which had been carefully gruaded against cross-pollination, were sown October 12, 1906, and 217 plants were raised, of which 188 lived to produce seed. 'These had the following composition: 98 were $B$. bursa-pastoris heteris, 36 B. bp.tenuis, 32 B. bp. rhomboidea, 13 B. bp. simplex, $5 \mathrm{~B}$. heegeri heteris, $1 \mathrm{~B}$. h. tenuis, $2 \mathrm{~B}$. h. rhomboidea, and $1 B$. h. simplex, giving, so far as the rosette-characters are concerned, a very close agreement with the ratio $9: 3: 3: 1$, but in the form of capsule showing a very great preponderance of the bursa-pastoris type.

0689.197: Seeds of 3 unguarded sibs of the parent of the last family were sown October 15, 1906, under this number. Of 2,014 offspring, 1,815 came to maturity, and were recorded as having the following composition: 1,032 were $B$. bursa-pastoris heteris, 331 B. bp.tenuis, 302 B. bp. rhomboidea, $78 \mathrm{~B}$. bp. simplex, $45 \mathrm{~B}$. heegeri heteris, $13 \mathrm{~B}$. h. temis, $13 \mathrm{~B}$. h. rhomboidea, and $1 \mathrm{~B}$. h. simplex (plates 3 and 4 ). Again there is a close agreement in the leaf-characters with the typical dihybrid ratio, $9: 3: 3: 1$, and a notable deficiency in the occurrence of the hecgeri type of capsizle.

0688.212: Seeds of this unguarded plant, which was a reciprocal of the parents of the last two families described, were sown October 12, 1906, and produced a large progeny, of which 621 unselected plants were potted for study and the rest discarded. Of these 621 plants, 537 reached maturity and were classified thus: 317 B. bursa-pastoris heteris, 102 B. bp. tenuis, 67 B.bp. rhomboidea, $21 \mathrm{~B}$. bp. simplex, $19 \mathrm{~B}$. heegeri heteris, $7 \mathrm{~B}$. h. tenuis, and $4 B$. h. rhomboidea, no $B$. h. simplex being observed. The same general relations of the rosette-characters and capsule-characters are obvious here as appeared in the other two $\mathrm{F}_{2}$ families described, but there is not quite as close agreement with the ratio $9: 3: 3: 1$ as in the other families, probably because this family became somewhat diseased and the distinguishing of the several types of rosette became consequently more difficult.

Reviewing the results of crossing Bursa bursa-pastoris simpler and $B$. heegeri, it is seen that the $F_{1}$ hybrids are essentially uniform, no matter in which direction the cross is made, and that the rosette in either case is of the heteris-rhomboidea type, owing to the incomplete clominance of heteris. while the capsule is always of the bursa-pastoris type. In li ${ }_{2}$ there appear the 4 types of rosette already described, in combination with each type of capsule. The rosette presented many instances of the best-cleveloped examples of the 4 described forms, particularly interesting being the fact 
that much better-developed heteris rosettes oceur in the $\mathrm{F}_{2}$ than are seen in pure-bred $B$. heegeri, though these heteris characteristics inust have come directly from the heegeri side of the cross.

The numerical results of these crosses may be tabulated thus:

$$
\begin{gathered}
\text { Bursa bp. simplex }(a b C) \times \text { Bursa h. heteris }(A B c) \\
(056) \quad(059) \\
\text { Bursa bp. heteris-rhomboidea } \\
(A B C a b c) \\
\text { (o6s8 and o689) }
\end{gathered}
$$

\begin{tabular}{|c|c|c|c|c|c|}
\hline & 06196 & 06197 & 06212 & Total. & Expected. \\
\hline \multicolumn{6}{|l|}{ Bursa-pastoris series $(C)$ : } \\
\hline 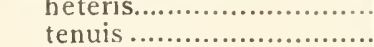 & $\begin{array}{l}98 \\
36\end{array}$ & $\begin{array}{r}1032 \\
331\end{array}$ & $\begin{array}{l}317 \\
102\end{array}$ & $\begin{array}{r}1447 \\
469\end{array}$ & $\begin{array}{r}1308 \\
456\end{array}$ \\
\hline rhomboidea.................. & 32 & 302 & 67 & 401 & 456 \\
\hline simplex................................. & 13 & $7^{8}$ & 21 & II 2 & 152 \\
\hline \multicolumn{6}{|l|}{ Heegeri series $(c)$ : } \\
\hline 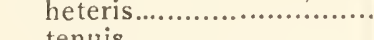 & 5 & 45 & 19 & 69 & 63 \\
\hline 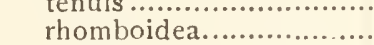 & $\begin{array}{l}1 \\
2\end{array}$ & $\begin{array}{l}13 \\
13\end{array}$ & $\begin{array}{l}7 \\
4\end{array}$ & $\begin{array}{l}21 \\
19\end{array}$ & $\begin{array}{r}21 \\
21\end{array}$ \\
\hline simplex. ........................... & 1 & I & 0 & 2 & 7 \\
\hline$C: C \ldots \ldots \ldots \ldots$ & 19.9: I & $=4.2: \mathrm{I}$ & I6.9: I & $21.9: 1$ & $3.0: \mathrm{I}$ \\
\hline
\end{tabular}

In this table the pedigree-numbers 06196 and 06197 represent families in which $B$. heegeri was the mother, while 06212 is the reciprocal cross. It will be seen that there is no essential difference between these two crosses. In the rosette-characters these numbers are very close to the Mendelian ratios, among both those having bursa-pastoris capsules and those having heegeri capsules, showing by these facts that the heegeri rosette has the same allelomorphic composition as the rosette of $B$. bursapastoris heteris, and that these rosette-characters are not coupled in any but a chance way with the form of the capsule. This independence of characters is thus a matter of great importance in the production of new elementary forms; for before this cross was made there existed, so far as is known, but one elementary species of $B$. heegeri, while out of the cross came 4 distinct elementary forms of this species.

While there is a perfect agreement with Mendelian ratios in rosettecharacters, the capsules give a surprising departure. The capsule-form is perfectly alternative; there has never appeared any intermediate condition in the plants in my cultures, and the "reversions" observed by SolmsLaubach and Laubert were pathological. As the bursa-pastoris type is dominant, simple Mendelian expectation would require the appearance of $1 \mathrm{~B}$. heegeri in every $4 \mathrm{~F}_{2}$ individuals. Out of 2,540 plants of the $\mathrm{F}_{2}$ generation observed, only 111 were $B$. heegeri, or approximately 1 in 23 . That this result should be consistently given in 3 different pedigrees representing reciprocal crosses adds greatly to the weight that is to be attached to 
it. The greatest frequency in any pedigree was 1 in nearly 18 , and the least frequency was 1 in 25 .*

I take the fact that the reciprocal crosses give similar ratios to indicate that the heegeri type of capsule is dependent 11 pon something carried by the germ-cells, and can not be a pathological conclition transmissible from mother to offspring somatically, a possibility which might acconnt for apparent heritability of characters in a self-fertilized line, but conld not well account for equal results in reciprocal crosses. Normal Mendelian phenomena are believed to rest pretty securely on the method of formation of the chromosomes during the reduction division, but no scheme of behavior occurs to me which would result in the production of a heegeri homozygote in only 1 indivichual in 23.

It is conceivable that the union of heegeri germ-cells in the hybrids forms a less successful combination than that into which the B. bursa-pastoris determiner enters, and that therefore fewer successful zygotes are formed by such unions, and it is also conceivable that only a small percentage of the $B$. heegeri succeeded in reaching sexual maturity, since in each of these families a considerable number of individuals failed to fruit, but the assumption that every individual that failed to seed was a $B$. heegeri would not nearly bring that form up to one-fourth of the entire progeny. There was no evidence in my cultures that $B$. heegeri is in any way inferior to $l$ ? bursa-pastoris or that it is any less likely to mature; neither have I observed any indication of the material lessening of fertility which would obtain if almost all of the heegeri homozygotes should fail in the initial stages of development.

*Dr. Correns tells me that he also made the cross between Bursa bursa-pastoris and $B$. heeger $i$ several years ago and likewise found a deficiency in the number of specimens having the heggeri type of capsule, but as his numbers were small he considered the deficiency due alone to the inadequate numbers. 


\section{DISCUSSION OF RESULTS.}

The occurrence of elementary species within the limits of recognized systematic or Linnean species is undoubtedly very general, as De Vries has maintained, and much of the ordinary conception as to the rariability of certain species is attributable to this fact. Thus in the specific case with which we are dealing here, Bursa is recognized by all taxonomists as exceedingly rariable, but each single biotype of Bursa is much less variable than the Linnean species taken as a whole, for when grown under farorable conditions there are certain characteristics which are fonnd in every individual of the given biotype which are not present in the members of other biotypes.

All the apparent evidence for the permanent change of species through selection finds a ready explanation on the assumption that the selection has merely eliminated eertain biotypes from the original mixture with which the selection started, thus leaving the theory that fluctuations are inherited or are capable of leading either directly or indirectly to the modification of any biotype wholly unsupported. This is not to say, of course, that such modification is impossible or that it does not take place, but merely that such a proposition must rest upon experience still to be gained.

There is some variation among the members of the single biotype. This variation is of the fluctuating kind by definition. The most usual variations of this kind are those which result from crowding; shading, poor soil, drought, the attacks of insects, or other conditions which decrease the rigor of the plants, the effect being to arrest differentiation in more or less jurenile stages of development. This feature has been rery troublesome at times in my cultures, since it is impossible under such circumstances to determine by inspection to which biotype a given specimen belongs. The breeding-test is the only method by which such a determination can be made and when many specimens have their distinguishing characteristics rendered latent in this way the labor, time, and patience required for complete classification become unduly increased.

This suppression of characteristics through fluctuation also stands in the way of the classification of specimens observed in nature in various habitats where they have grown under different conditions which are in a large degree unknown, and on this acconnt there can be no question as to the advisability of retaining the Linnean names in practical taxonomy for the designation of such complex groups of biotypes. Workers in all other botanical fields must bear in mind, however, that conclusions reached with one biotype may not hold in some other biotype of the same Linnean species.

The demonstration of the elementary character of these biotypes of Bursa is made complete by the fact that they Mendelize on being crossed, for such 
behavior can only rest upon the presence of an internal factor, of whatever nature, capable of independent movement at the time of the reductiondivision in the formation of the germ-eclls. It may be assumed, perhaps, that this factor or determiner is incapable of division at the time of the reduction-division; but however it is explained, the result is the produetion of certain indivicluals (homozygotes) which produee germ-eells all of one kind with respect to a particular character and which must therefore breed true with respect to that character, and other individuals (het erozygotes) which prodnce germ-eells of two kinds with respect to the same character and which consequently ean not breed true. Individuals which possess the same characteristics and which are homozygous with respect to all of these characteristics, collectively form a biotype ; henee the statement that the Mendelian inheritance of the several forms of Bursa demonstrate beyond a possible question that they are distinct biotypes. I will not be inderstood, however, to imply that biotypes must Mendelize on being crossed, for many are known which do not, as, for example, most of the varions biotypes of Oenothera.

The importance of Mendel's discoveries for our eonception of the significance of different kinds of variations in the evolution of plants and animals is now generally recognized. It is seen that in the presence of Mendelian inheritance the "swamping" of a new eharacter by erossing with the parent form does not take place, and that the very kind of variation which was supposed to be swamped by erossing is just the kind which is most certain to be preserved. The new form may completely disappear when crossed, but it is only hidden momentarily, not destroyed. Thus when Bursa heegeri erosses with its parent Bursa bursa-pastoris, all of the offspring are indistinguishable from the latter, but in the next generation a portion of the offspring are just as typical $B$. heegeri as the original specimen, and no transitional forms oceur. If the dominance of the triangular capsules were incomplete there would be some transitional stages, but this would not modify the situation in any essential manner, as there would still be just as many typical homozygotes as before which would breed true whenever they chanced to be fertilized by their own kind, and if fertilized again by the parent form they would form heterozygotes which would produce as large proportion of typical $B$. heegeri as was produced by the heterozygotes of the previous generations.

I have shown elsewhere (Shu11, 1907a) that this capacity of a mutant to disappear upon crossing with its parent may be a great adrantage in the struggle for existence under certain conditions, and if we grant that erolution is in the main retrogressive (Shu11, 1907b), the new forms will be generally recessive to the parent and will thus be in position to profit by whatever advantage recessiveness gives. From the data I have as to the geographic distribution of the several biotypes of $B$. bursa-pastoris it 
appears that $B . b p$. heteris is much more generally distributed than any of the others, and this favors the view that this is the original type from which B. bp. rhomboidea, tenuis, and simplex were derived by retrogressive mutation. Reeessiveness of the last thrce to the first is probably rarely if ever any advantage to them, however, since all of these forms appear to be about equally adapted to the range of habitats in which they grow.

Mendelian inheritance also has an important bearing upon the distribution of the various biotypes of a species; for the transportation of a single hybrid seed may carry all of the biotypes which are related to one another in the Mendelian way, however many there may be, ${ }^{*}$ and two pure-bred seeds landed in the same vicinity may lead to the same result. Thus any seed of $B$. bp. heteris which has been produced by pollination with pollen from $B$. $b p$. simplex, or wice versa, or any seed of $B$. $b p$. rhomboidca that has resulted from pollination with pollen from $B$. bp. tenuis, or vice versa, will give rise to a progeny in the $\mathrm{F}_{2}$ which will inelude all four of these biotypes, and self-evidently two pure-bred seeds representing either of these two pairs of elementary species will earry the capacity to produce the same four types in the third generation from the time they find themselves in juxtaposition in a new locality.

The same principle wonld hold if there were 3 pairs of Mendelian characters involved, but then 8 biotypes might be earried by a single hybrid seed. This situation would be realized by the material dealt with in this paper if a single seed of $B$. bp. simplex pollinated by $B$. heegeri, or sice versa, were taken to a new loeality. Transcau (1907) has pointed out how on the same principle any number of biotypes might be introduced by means of a single pollen grain into a new loeality where a single biotype had existed before, and to which heary seeds might find mueh greater diffculty in being transported.

Still another important effect of Mendelian inheritance in the promotion of organic crolution is brought out by my crosses between $B$. bursa-pastoris and $B$. heegeri, namely, the production of parallel series of biotypes in nearly related speeies. Up to the time this cross was made B. heegeri was known only in the heteris form, but among the hybrid offspring were 4 distinct pure-breeding biotypes of $B$. heegeri. It is thus seen that the single mutation by which $B$. heegeri originated from $B$. bursa-pastoris doubled the number of possible biotypes of Bursa in the world, provided all such other biotypes behave as do the four under discussion in this paper.

It may be added that the faets here shown that the rosette of $B$. heegeri represents the same Mendelian units that are present in $B$. bursa-pastoris and that there is only a single fundamental difference between these two species is the best possible proof of the origin of B. heegeri from B. bursa-

*Except in the presence of "spurious allelomorphism." 
pastoris, despite the considerable differences of general aspect both of foliage and of inflorescence. It is possible that these differences of aspect may rest upon the presence of still other units which have not been taken into account in these studies, but if such should prove to be the case, the genera1 conclusions must be the same.

I wish to acknowledge my appreciation of the facilities which have been placed at my disposal by the Station for Experimental Erolution of the Carnegie Institution of Washington, and of the faithfulness of my assistants, who have greatly aided me with the technical side of this investigation. My thanks are due to my brother, J. Marion Shu1l, dendrological artist of the United States Forest Service, for the drawings reproduced in figs. 1 to 23. I particularly desire to acknowledge my indebtedness to Dr. E. N. Transeau, in whose charge the cultures of $B$. bursa-pastoris $\times$ heegeri were left during my absence in California in the spring of 1907. Much of the work of grouping the $\mathrm{F}_{2}$ into the appropriate classes was done by him.

\section{SUMMARY.}

(1) Bursa (Capsella) bursa-pastoris is a composite species made up of at least 4 and possibly many distinct elementary species or biotypes; 4 of these are described under the names Bursa bursa-pastoris heteris, B. bp. tenuis, B. bp. rhomboidea, and B. bp. simplex. Except for the suppression of characteristics dive to bad treatment, the fluctuations of these forms are slight.

(2) These 4 biotypes cross readily, giving in each case a 11niform $\mathrm{F}_{1}$ and typical Mendelian splitting in $\mathrm{F}_{2}$. They are differentiated from each other by 2 init-characters, namely, elongated primary lobes of the climax-leaves and a rounded secondary lobe in the distal axil of the primary lobes. When $B . b p$. heteris is crossed with $B$. bp. simplex, and when $B$. bp. tenuis is crossed with $B . b p$. rhomboidea, the $\mathrm{F}_{1}$ is intermediate between $B$. $b p$. heteris and $B . b p$. rhomboidea becanse of the imperfect dominance of $B$. bp. heteris, and the $\mathrm{F}_{2}$ consists of heteris, temis, rhomboidea, and simplex. in the ratio $9: 3: 3: 1$.

(3) The sterile or nearly sterile plants supposed by Almquist to be hybrids were probably not hybrids, as similar forms were noted in guarded cultures, and in all of my hybrids no decrease in fertility was apparent.

(4) Bursa heegeri differs from B. bursa-pastoris in the aspect of its rosette and inflorescence, but most notably in the form of the capsule. Its climax-leaves are of the same general type as those of $B$. bp. heteris.

(5) Bursa heegeri may be readily crossed with $B$. bursa-pastoris. When crossed with $B$. bp. simplex, an $F_{1}$ is obtained which more nearly resembles $B$. heegeri in rosette characters, but has the capsule-form of $B$. bursapastoris. In $\mathrm{F}_{2}$ there appear the 4 forms of rosette already mentioned, in 
the ratio $9: 3: 3: 1$, in association with each type of capsule. The ratio of the bursa-pastoris type of capsule to the heegeri type is about $22: 1$.

(6) Bursa heegeri possesses the same :1nit-characteristics in the rosette as $B$. bp. heteris, which serves to further confirm its direct derivation from that species.

(7) The fact that the various forms of Bursa show Mendelian inheritance on crossing is conclusive proof that they are distinct biotypes, notwithstanding the fact that their distinguishing characteristics are readily rendered latent by flutctiation.

(8) In the presence of Mendelian inheritance the swamping of a new characteristic does not take place, and the kind of variation which has been strpposed to be swamped by crossing is just the kind that is most certain to be preserved.

(9) The recessiveness of a newly arisen form is to be considered an advantage in its struggle with the parent, whenever the former is in any way less adapted to its environment than the latter. This principle apparently has no bearing mpon these biotypes of Bursa, however, as all appear to be equally adapted to the range of habitats in which they live.

(10) Bursa bursa-pastoris hetcris appears to have a more greneral distribution than any of the other forms. This is probably the primitive type from which the other biotypes have been derived by retrogressive mutation.

(11) Mendelian inheritance also assists in the distribution of the various biotypes, since a single hybrid seed or two pure-bred seeds may carry into a new locality as many distinct biotypes as are related to each other by Mendelian characters. A single pollen-grain may do the same provided one biotype is already present in the locality in question.

(12) Crosses between nearly related species may give rise to parallel series of biotypes in the two species concerned. Before my crosses between Bursa hegeri and B. bursa-pastoris were made, the former was known only in 1 form, but out of the crosses came 4 distinct biotypes of $B$. heegeri, corresponding with the 4 biotypes which have been demonstrated in $B$. bursa-pastoris.

(13) Each mutation which results in the appearance of a new Mendelian nnit-character doubles the possible number of Mendelian biotypes belonging to the species in question, however nttmerous they may be already, except as limited by "spurious allelomorphism.",

Station for Experimental Evolution, July 11, 1908. 


\section{LITERATURE CITED.}

AlMQUist, E.

1907. Studien iiber die Capsella bursapastoris (L.). Acta Horti Bergiani, 4:

DE VRIES, H. No. 6, pp. 92, figs 66, May 15, 1907.

190I. Die Mutationstheorie. I. Die Entstehung der Arten durch Mutation. pp. xii +64 S, pls. 8, figs. ISI, 1901 . Leipzig. (See pp. 477-478.)

1905. Species and varieties: their origin by mutation. pl. xviii $+847,1905$. Chicago. (See pp. $5^{82-5^{8}} 4$.)

$\mathrm{HuS}, \mathrm{H}$.

19o8. Fasciations of known causation. Amer. Nat., 42: 8I-97, 2 figs., Feb., 1908.

LAUBERT, R.

1905. Notizen über Capsella heegeri Solms. Verh. Bot. Vereins Provinz Branden-

Lotsy, J. P. burg, 47: 197-199, 4 figs., 1905.

1906. Vorlesungen über Deszendenztheorien mit besonderer Berücksichtigung der botanischen Seite der Frage. Erster Teil, pp. $3^{8} 4$, pls. 2, figs. 124, 1906, Jena. (See pp. 179-181.)

NolL, F.

1907. Ueber eine Heegeri-ähnliche Form der Capsella bursa-pastoris Moench. Sitzungsber. Niederrhein. Gesells. Nat.-u. Heilkunde, 5 pp., 1907, Bonn.

PotoniE, H.

1906. Capsella heegeri eine pathologische Erscheinung mit atavistischen Momen-

SHULL, G. H. ten? Naturwis. Wochenschr., 21 (n. s., 5): 788-791, 2 figs., Dec. 9, 1906.

1907a. Elementary species and hybrids of Bursa. Science, n. s., 25: 590-591, Apr.

$1907 b$. The significance of latent characters. Science, n. s., 25: 792-794, May 17, 1907.

Igo8. A new Mendelian ratio and several types of latency. Amer. Nat., 42: 433451 , July, r 908 .

Solms-LaUBACH, H.

Igoo. Cruciferienstudien. I. Capsella heegeri Solms eine neuentstandene Form

Transeau, E. N. der deutschen Flora. Bot. Zeit., 58: 167-190, pl. vii, 1900.

1907. Hybridization a factor in migration and competition. Science, n. s., 25: 269-270, Feb. 15,1907 . 



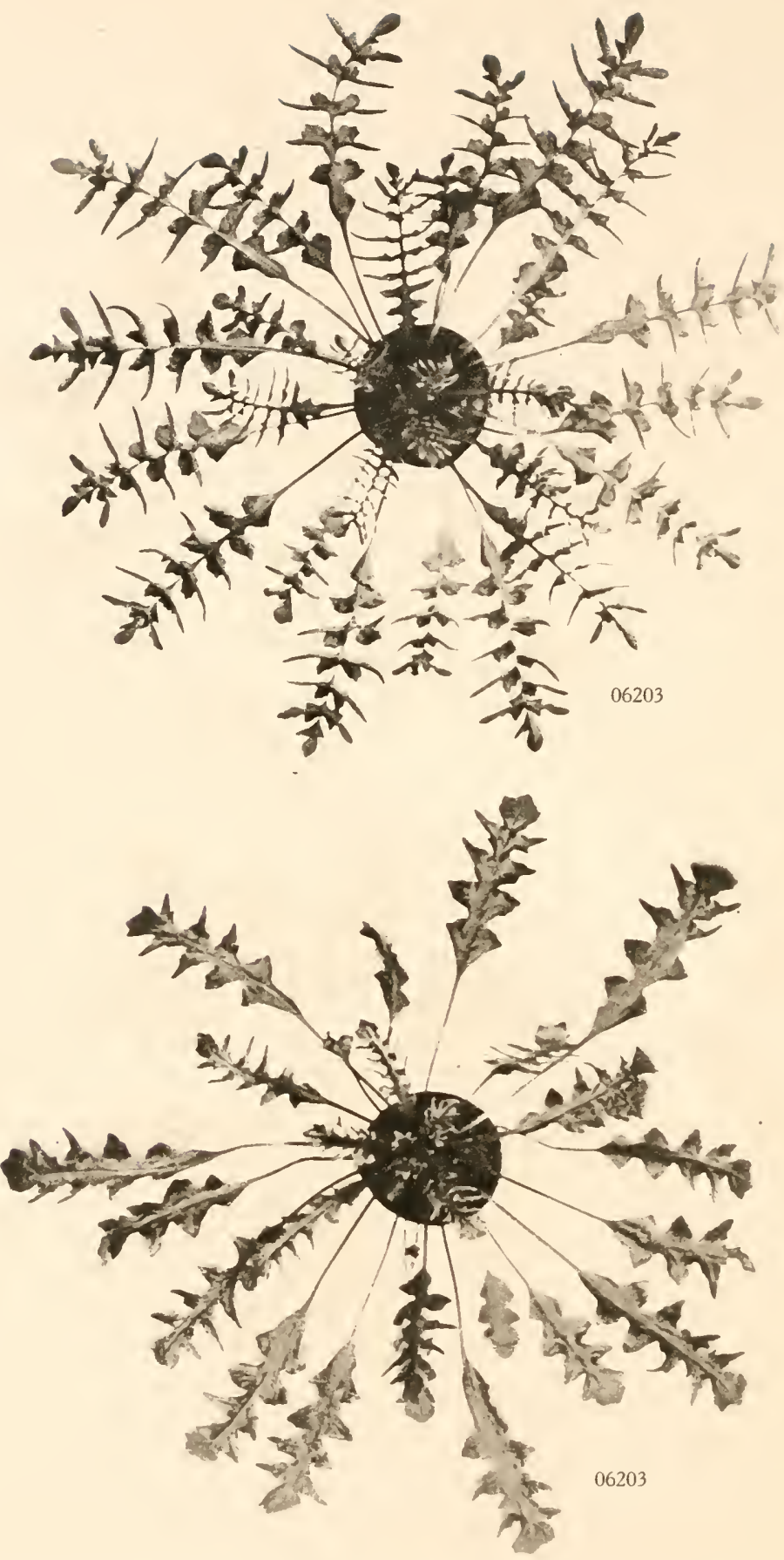

Fig. 1. - Bursa bursa-pastoris heteris. Dominant form from the $F_{2}$ of an artificial cross of B. bp. heteris $\times$ tenuis. The grandparents were sibs of the plants shown in text-figures 4 and 9 .

Fig. 2. - Bursa bursa-pastoris tenuis. Recessive form from the same family as the plant shown in figure 1 . 

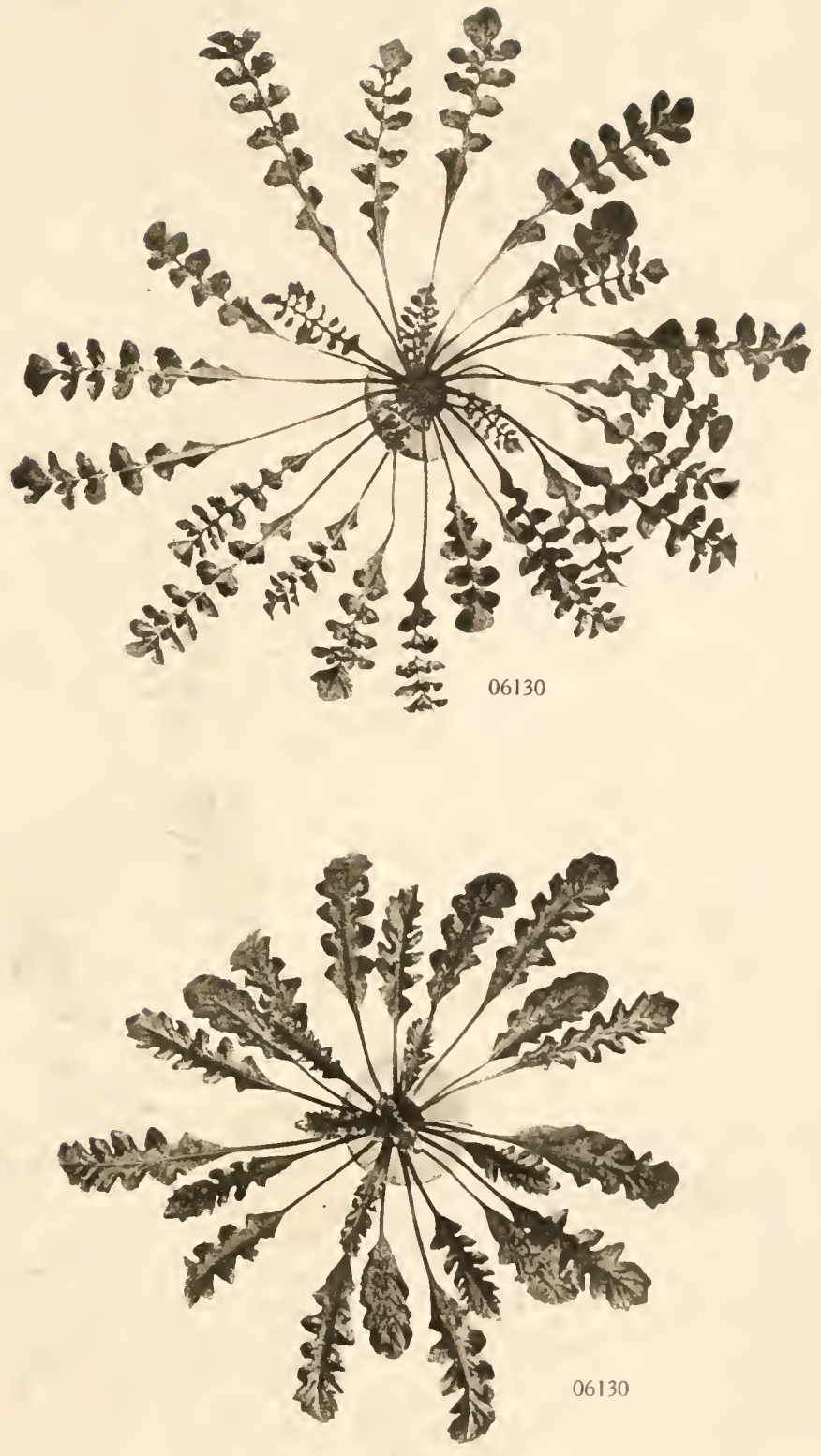

Fig. 1. - Bursa bursa-pastoris rhomboidea. Dominant form in the $F_{2}$ of a hybrid family representing the natural cross, B. bp. simplex $\times$ rhomboidea. The parent was a sib of the plant shown in text-figure 20 and had the same characteristics.

Fig. 2. - Bursa bursa-pastoris simplex. Recessive form in the same hybrid family whose dominant form is represented by figure 1 . 



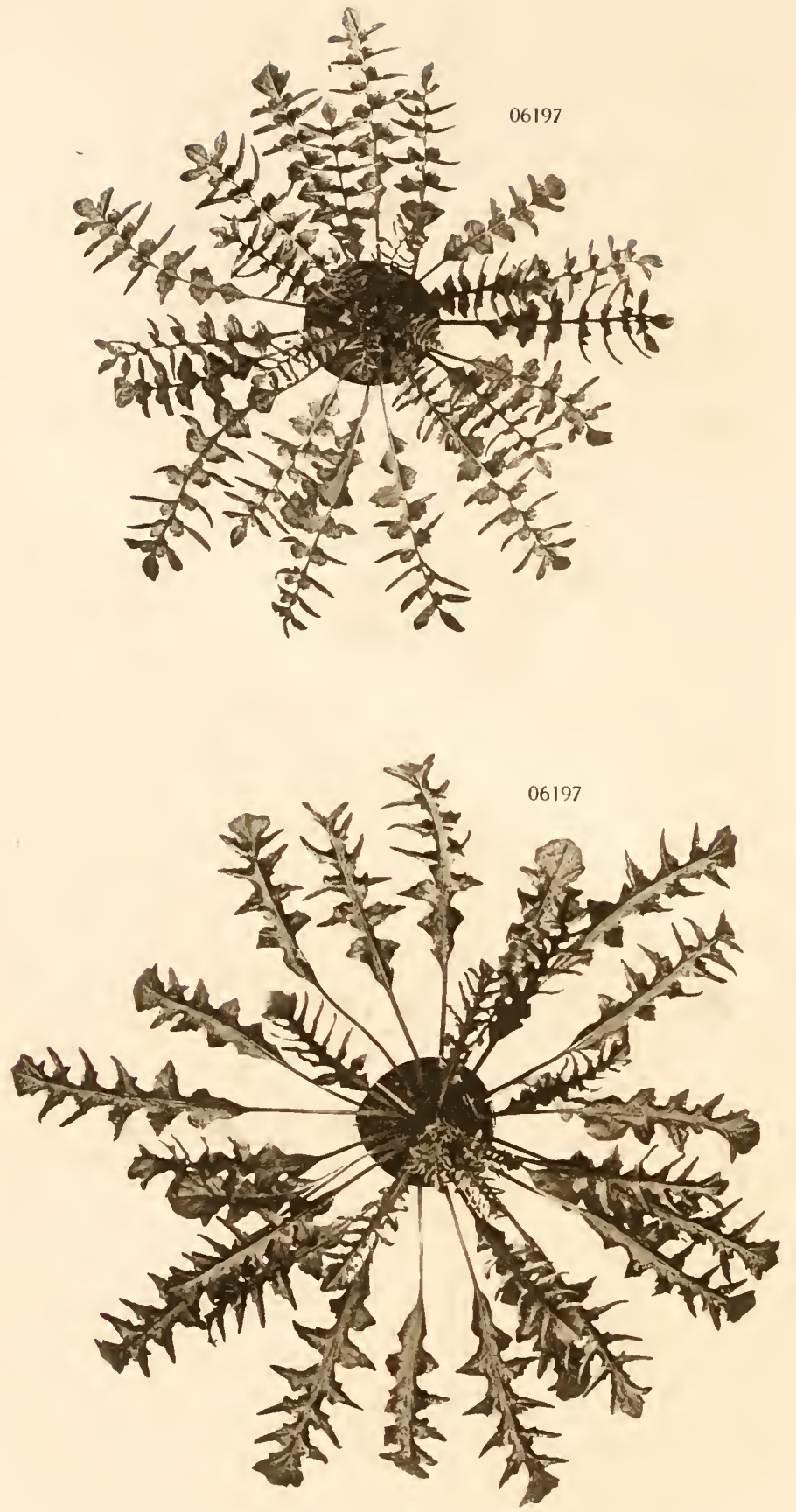

Fig. 1. Bursa heegeri $\times$ bursa-pastoris simplex. A heteris rosette in the $F_{2}$.

Fig. 2. Bursa heegeri $\times$ bursa-pastoris simplex. A tenuis rosette in the $F_{2}$. 

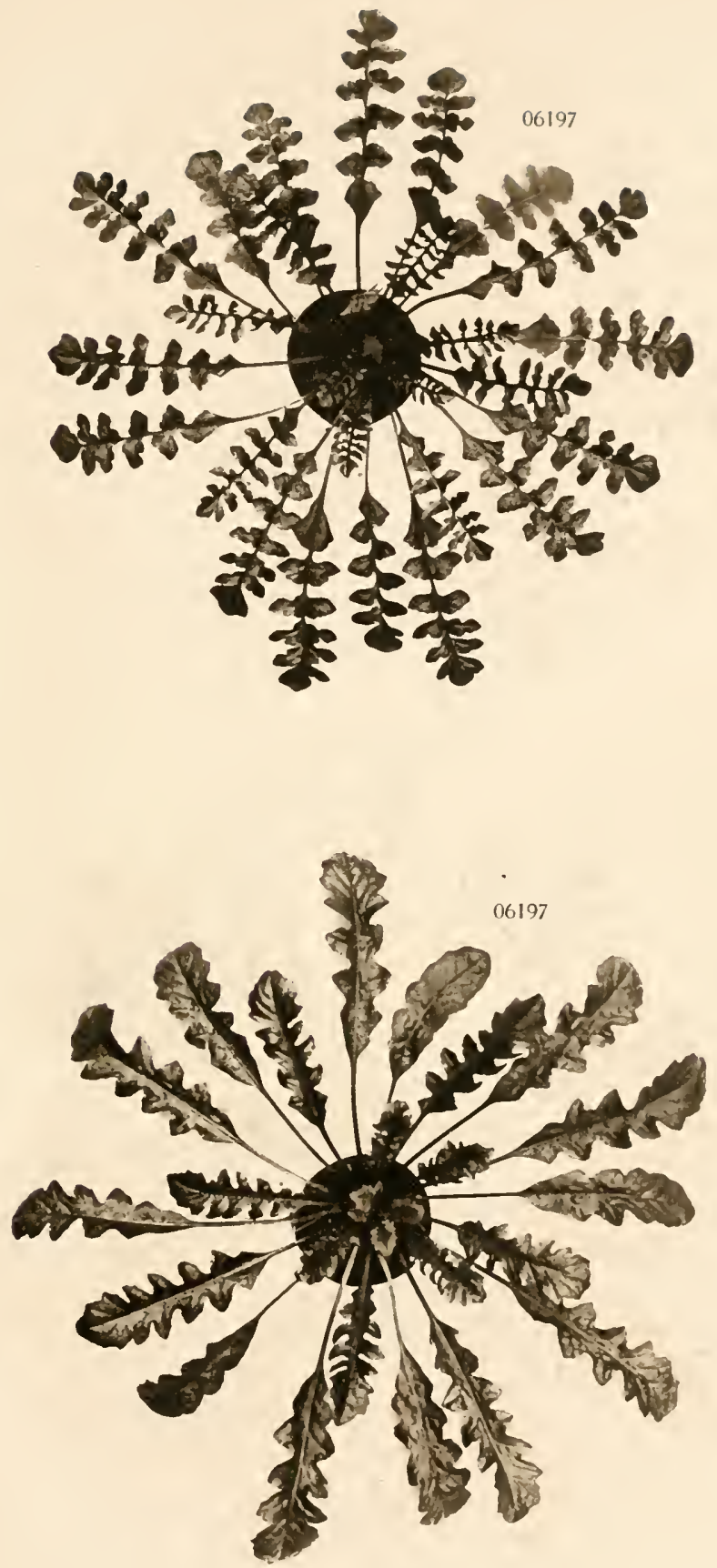

Fig. I. Bursa heegeri $\times$ bursa-pastoris simplex. A rhomboidea rosette in the $F_{2}$.

Fig. 2. Bursa heegeri $\times$ bursa-pastoris simplex. A simplex rosette in the $F_{2}$. 
2 
- 




North Carolina State University Libraries QH406.S54

BURSA BURSA.P

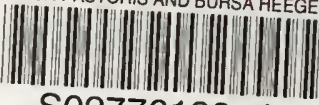

S02776138 J 